Karl-Franzens Universität Graz

Technische Universität Graz

Medizinische Universität Graz

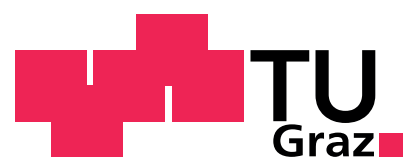

\title{
Vortex control in channel flows using translation invariant cost functionals
}

\author{
Henry Kasumba Karl Kunisch
}


SFB sponsors:

- Austrian Science Fund (FWF)

- University of Graz

- Graz University of Technology

- Medical University of Graz

- Government of Styria

- City of Graz

Das Land

Steiermark

Stadt $\mathbf{G} \mathbf{R}$ A Z $\mathbf{Z}$ Wissenschaft 


\title{
Vortex control in channel flows using translation invariant cost functionals
}

\author{
H. Kasumba* and K. Kunisch ${ }^{\dagger}$
}

\begin{abstract}
The use of translation invariant cost functionals for the reduction of vortices in the context of shape optimization of fluid flow domain is investigated. Analytical expressions for the shape design sensitivity involving different cost functionals are derived. Channel flow problems with a bump and an obstacle as possible control boundaries are taken as test examples. Numerical results are provided in various graphical forms for relatively low Reynolds numbers. Striking differences are found for the optimal shapes corresponding to the different cost functionals, which constitute different quantification of a vortex.
\end{abstract}

Key words: Vortex quantification, Shape optimization, Navier-Stokes equation.

\section{Introduction}

An important topic in the field of optimal control of partial differential equations is the choice of an appropriate cost functional to quantify the control objective. This functional depends on the state variables $(\mathbf{u}, p)$, where $\mathbf{u}$ and $p$ are the velocity and pressure of the fluid, respectively, and in our case on the control parameters describing the shape of the domain. Typical cost functionals for vortex reduction, are based on tracking-type functionals or minimization of the curl of the velocity field, [10],[1], i.e.,

$$
J_{1}(\mathbf{u})=\frac{1}{2} \int_{\tilde{\Omega}}\left|\mathbf{u}(x)-\mathbf{u}_{d}(x)\right|^{2} d x, J_{2}(\mathbf{u})=\frac{1}{2} \int_{\tilde{\Omega}}|\operatorname{curl} \mathbf{u}(x)|^{2} d x,
$$

where $\tilde{\Omega}$ describes the subset of $\Omega$ over which vortex reduction is desired and $\mathbf{u}_{d}$ stands for a given desired flow field which contains some of the expected features of the controlled flow field without the undesired vortices. The tracking type cost functional $J_{1}$ has the disadvantage that it does not attempt to quantify the vortices in the flow in terms of intrinsic properties of the velocity field $\mathbf{u}$ or pressure $p$ [16]. Moreover, it has the disadvantage that it is not invariant under changes of frames which move at a constant speed relative to each other. Functionals which allow such a property are referred to as Galilean invariant. Turning to the cost functional $J_{2}$, it is important to note that the vorticity, curl $\mathbf{u}(x)$, is Galilean invariant (see, e.g., [20, Chapter 5] for more details). By using this functional, vortices in the flow can be thought of as regions of high vorticity magnitude. However, there is no universal threshold over which the magnitude of the vorticity is to be considered high [12]. More alarmingly, the vorticity magnitude (|curlu|) may also be high in parallel shear flows where no vortices are present [17].

\footnotetext{
* Johann Radon Institute for Computational and Applied Mathematics, Austrian Academy of Sciences, Altenbergerstraße 69, A-4040 Linz, Austria, email: henry.kasumba@ oeaw.at.

${ }^{\dagger}$ Institut für Mathematik und Wissenschaftliches Rechnen, Karl-Franzens-Universität Graz, Heinrichstraße 36, A-8010 Graz, Austria; email: karl.kunisch@uni-graz.at.
} 
A literature study suggests that in fluid dynamics and dynamics systems theory, the quantification of a vortex is still an issue that is not completely settled. Research in [3], [6], suggests that in 2D, vortex cores are related to regions with complex eigenvalues of the velocity gradient tensor $\nabla \mathbf{u}$. This vortex definition is Galilean invariant [11], [17]. From the linear-dynamic system point of view [18], this definition suggests that a local streamline pattern is closed or spirals in a reference frame moving with the particle. In 2D, eigenvalues of $\nabla \mathbf{u}$ are complex if $\operatorname{det} \nabla \mathbf{u}>0$ [17], and this suggests to choose

$$
\int_{\tilde{\Omega}} \max (0, \operatorname{det} \nabla \mathbf{u}(x)) d x,
$$

as cost functional [16]. Since this cost functional is based on a Galilean invariant vortex definition, it is a Galilean invariant cost functional. From the mathematical point of view, it penalizes the complex eigenvalues of the velocity gradient tensor $\nabla \mathbf{u}(x)$ which are responsible for the swirling motion. However, due to the max-operation, the cost functional in (2) is not differentiable and hence,we introduce the smoothing function $g_{3} \in C^{2}(\mathbb{R})$ defined, e.g., by

$$
g_{3}(t)=\left\{\begin{array}{cc}
0, & t \leq 0 \\
t^{3} /\left(t^{2}+1\right), & t>0
\end{array}\right.
$$

such that

$$
J_{3}(u)=\int_{\tilde{\Omega}} g_{3}(\operatorname{det} \nabla \mathbf{u}(x)) d x .
$$

A first step towards investigating Galilean invariant cost-functionals for optimal vortex control in fluids was carried out for a driven-cavity problem in [16], and later for a flow around an obstacle in [19]. In [19], striking differences were found for the optimal controls corresponding to the three different cost functionals expressed in (1)-(3). In the current work the authors systematically analyze optimal shapes corresponding to the minimization of functionals (1-3). A comparison among the three cost functionals is made using three test problems. The first two test problems consist in minimization of vortices in flows in a channel with a bump and an obstacle respectively. The third test case consists in consideration of an irrotational flow in a channel with a bump. For the purpose of minimization, a gradient type method is used. It relies on the characterization of the shape gradients for all three functionals. The continuous formulations are discretized and numerical algorithms for solving the discrete shape optimization problems are developed and implemented.

The remainder of this paper is organized as follows. Section 2 describes the setting of the state and optimization problems. Section 3 introduces the existence analysis of the state and optimization problems. The sensitivity analysis of the optimization problems is given in Section 4. In Section 5, the numerical algorithm used to realize the optimization problems is given. Numerical examples that support the theoretical results are presented and the conclusions of this work are finally drawn. 


\section{Setting of the problem}

\subsection{State problem}

Let $\Omega \subset \mathbb{R}^{2}$ with a sufficiently piecewise regular boundary $\partial \Omega=\Gamma$. Suppose that an incompressible viscous flow occupies $\Omega$, and that the state equation for the flow is given by the following system of Navier-Stokes equations in non-dimensional form:

$$
\left\{\begin{array}{l}
-v \Delta \mathbf{u}+(\mathbf{u} \cdot \nabla) \mathbf{u}+\nabla p=\mathbf{f} \text { in } \Omega, \\
\operatorname{div} \mathbf{u}=0 \text { in } \Omega .
\end{array}\right.
$$

Here $\mathbf{u}=\left(u_{1}, u_{2}\right)$ is the velocity field, $p$ the pressure, $v$ the kinematic viscosity of the fluid ( $v=\frac{1}{R e}>0$, where $R e$ is the Reynolds number), and $\mathbf{f}$ the density of external forces. The non-linear term $(\mathbf{u} \cdot \nabla) \mathbf{u}$ in (4) is a symbolic notation for the vector $\left(u_{1} \frac{\partial u_{1}}{\partial x_{1}}+\right.$ $\left.u_{2} \frac{\partial u_{1}}{\partial x_{2}}, u_{1} \frac{\partial u_{2}}{\partial x_{1}}+u_{2} \frac{\partial u_{2}}{\partial x_{2}}\right)$. In order to make (4) well-posed, we have to impose appropriate boundary conditions. In this work, different boundary conditions posed on the domains shown in Figure (1(a)-(b)) are considered, giving rise to 3 different test problems. In

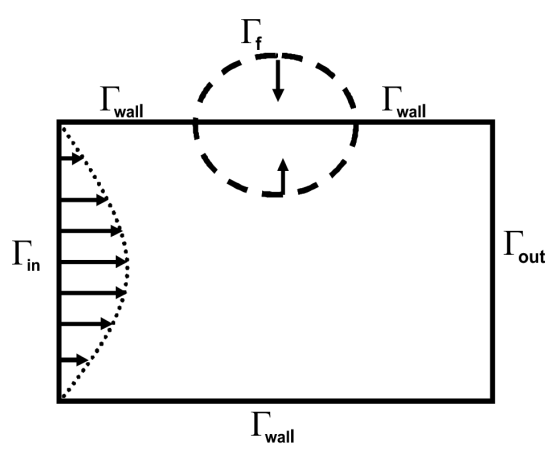

(a) Problem 1

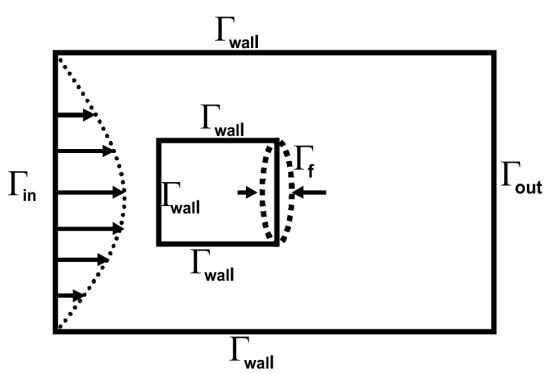

(b) Problem 2

Figure 1: Domains for test problems

the first test case, a parallel flow in a channel with a bump (Figure 1 a) is considered. In this case, the following boundary conditions

$$
\left\{\begin{array}{l}
\mathbf{u}=\mathbf{g} \text { on } \Gamma_{i n}, \\
\mathbf{u}=\mathbf{0} \text { on } \Gamma_{w} \cup \Gamma_{f}, \\
-p \mathbf{n}+v \frac{\partial \mathbf{u}}{\partial \mathbf{n}}=0 \text { on } \Gamma_{\text {out }},
\end{array}\right.
$$

are imposed. Here the vector $\mathbf{g}$ is a given velocity at the inflow $\Gamma_{i n}$. At the outflow $\Gamma_{\text {out }}$, a natural "do-nothing" boundary condition proposed in [15] is imposed. Along the fixed wall, $\Gamma_{w}$, and the control boundary, $\Gamma_{f}$, of the channel, the velocity vanishes. In the second test problem, a parallel flow in a channel with an obstacle (Figure $1 \mathrm{~b}$ ) is considered. Again the boundary conditions given in (5) are imposed. In the third test problem, an irrotational flow in a channel with a bump (Figure 1 a) is considered. In this case, the boundary condition is

$$
\mathbf{u}=\mathbf{g} \text { on } \Gamma=\Gamma_{\text {in }} \cup \Gamma_{w} \cup \Gamma_{f} \cup \Gamma_{\text {out }},
$$


where the vector $\mathbf{g}$ is a given velocity on the boundary $\Gamma=\Gamma_{i n} \cup \Gamma_{w} \cup \Gamma_{f} \cup \Gamma_{\text {out }}$.

For compatibility reasons, $\mathbf{g}$ in (6) must satisfy

$$
\int_{\Gamma} \mathbf{g} \cdot \mathbf{n} d s=0
$$

where $\mathbf{n}$ is the unit normal vector along the boundary $\Gamma$.

In all the test cases, the boundary $\Gamma_{f}$ is used as a control boundary by means of which the shape of $\Omega$ will be governed.

\subsection{Optimization problems}

Our goal is to find "an optimal" $\Gamma_{f}$ by minimizing the cost functionals in (1-3) which depend on $(\Omega, \mathbf{u})$. We let $\Gamma_{f}$ be described as a graph represented by the curve $\alpha$ : $[a, b] \mapsto \mathbb{R}$. Consequently, the problem of finding "an optimal" $\Gamma_{f}$ is equivalent to the one of finding an optimal control $\alpha$ over a set of admissible controls $\mathscr{U}_{a d}$ to be specified later on. Let $\mathscr{G}$ be the graph of the control-to-state (generally multi-valued) mapping :

$$
\mathscr{G}:=\left\{\{\alpha, \mathbf{u}, p\} ; \alpha \in \mathscr{U}_{a d},\{\mathbf{u}, p\} \text { is a weak solution of (4)-(5,6) }\right\} .
$$

The optimization problem can be written in the following form:

$$
\left\{\begin{array}{l}
\text { Find }\left\{\alpha^{*}, \mathbf{u}^{*}, p^{*}\right\} \in \mathscr{G} \text { such that } \\
J\left(\Omega\left(\alpha^{*}\right), \mathbf{u}(\alpha *)\right) \leq J(\Omega(\alpha), \mathbf{u}(\alpha)) \text { for all }\{\alpha, \mathbf{u}, p\} \in \mathscr{G} .
\end{array}\right.
$$

To describe $\mathscr{U}_{a d}$, we let $\Gamma_{f}$ be described as a graph represented by the curve $\alpha:[a, b] \mapsto \mathbb{R}$ which we assume to be given by

$$
\Gamma_{f}(\alpha)=\left\{\left(x_{1}, x_{2}\right): x_{1} \in[a, b], x_{2}=\alpha\left(x_{1}\right)\right\},
$$

for problem 1 and

$$
\Gamma_{f}(\alpha)=\left\{\left(x_{1}, x_{2}\right): x_{1}=\alpha\left(x_{2}\right), x_{2} \in[d, e]\right\},
$$

for problem 2, where $a, b, d$, and $e$ are given constants. (see Figure 2).

Consequently, one may define the admissible family of curves defining $\Gamma_{f}(\alpha)$ for problem 1 as follows:

$$
\begin{array}{r}
\mathscr{U}_{a d}=\left\{\alpha \in C^{0,1}([a, b]) \mid 0<\alpha_{\text {min }} \leq \alpha\left(x_{1}\right) \leq \alpha_{\text {max }},\right. \\
\left.\alpha(a)=\alpha_{0}, \alpha(b)=\alpha_{1},\left|\alpha^{\prime}\right| \leq L_{1}, \text { a.e in }(a, b)\right\},
\end{array}
$$

and

$$
\begin{array}{r}
\mathscr{U}_{a d}=\left\{\alpha \in C^{0,1}([d, e]) \mid 0<\alpha_{\min } \leq \alpha\left(x_{2}\right) \leq \alpha_{\max },\right. \\
\left.\alpha(d)=\alpha_{0}, \alpha(e)=\alpha_{1},\left|\alpha^{\prime}\right| \leq L_{2}, \text { a.e in }(d, e)\right\},
\end{array}
$$

for problem 2, where $L_{1}, L_{2}, a, b, d, e, \alpha_{\min }, \alpha_{\max }$ are given constants such that $\mathscr{U}_{a d}$ is non-empty. 


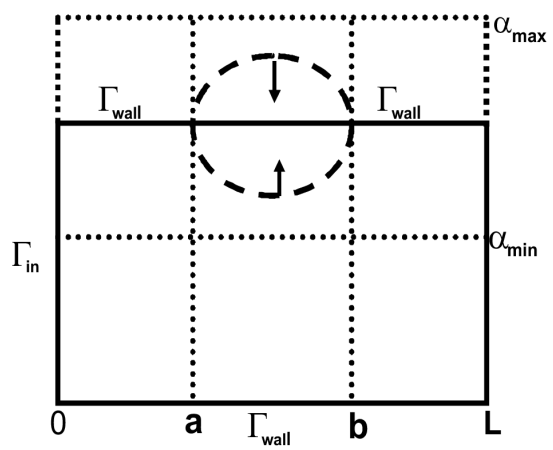

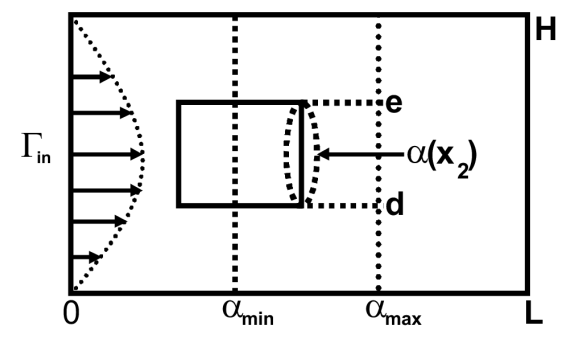

(b) Problem 2

(a) Problem 1

Figure 2: Geometric constraints for test problems

\subsection{Notation}

Here we collect some notations and definitions that we need in our subsequent discussion. Throughout the paper, we restrict ourselves to the two dimensional case. Vector-valued functions are indicated by bold letters. An element in $\mathbb{R}^{2}$, is denoted by $x=\left(x_{1}, x_{2}\right)$ with norm $|x|_{\mathbb{R}^{2}}=\left(\sum_{j=1}^{2} x_{j}^{2}\right)^{1 / 2}$. Two notations for the inner product in $\mathbb{R}^{2}$ shall be used, namely $(x, y)$ and $x \cdot y$ respectively. The latter shall be used in case of nested inner products. For a vector valued function $\mathbf{u}$, the gradient of $\mathbf{u}$, denoted by $\nabla \mathbf{u}$, is a second order tensor defined as $\nabla \mathbf{u}:=\left(\frac{\partial u_{j}}{\partial x_{i}}\right)_{i, j=1,2}$, while the Jacobian of $\mathbf{u}$, denoted by $D \mathbf{u}$, is the transpose of the gradient. The curl of a vector field $\mathbf{u}=\left(u_{1}, u_{2}\right) \in \mathbb{R}^{2}$, denoted by curl $\mathbf{u}$, is defined as curl $\mathbf{u}:=\frac{\partial u_{2}}{\partial x_{1}}-\frac{\partial u_{1}}{\partial x_{2}}$, while the curl of a scalar field $u$, denoted by $\operatorname{curl} u$, is defined as $\operatorname{curl} u:=\left(\frac{\partial u}{\partial x_{2}},-\frac{\partial u}{\partial x_{1}}\right)$. The determinant of the velocity gradient tensor of a vector field $\mathbf{u}=\left(u_{1}, u_{2}\right) \in \mathbb{R}^{2}$, denoted by $\operatorname{det} \nabla \mathbf{u}(x)$, is defined as $\operatorname{det} \nabla \mathbf{u}(x):=\frac{\partial u_{1}}{\partial x_{1}} \frac{\partial u_{2}}{\partial x_{2}}-\frac{\partial u_{2}}{\partial x_{1}} \frac{\partial u_{1}}{\partial x_{2}}$. Furthermore, we define the tensor scalar product denoted by $\nabla \mathbf{u}: \nabla \boldsymbol{\psi}$ by

$$
\nabla \mathbf{u}: \nabla \boldsymbol{\psi}:=\left(\sum_{i, j=1}^{d} \frac{\partial u_{j}}{\partial x_{i}} \frac{\partial v_{j}}{\partial x_{i}}\right) \in \mathbb{R} .
$$

We denote by $H^{m}(\mathscr{S}), m \in \mathbb{R}$, the standard Sobolev space of order $m$ defined by

$$
H^{m}(\mathscr{S}):=\left\{u \in L^{2}(\mathscr{S}) \mid D^{\alpha} u \in L^{2}(\mathscr{S}), \text { for } 0 \leq|\alpha| \leq m\right\},
$$

where $D^{\alpha}$ is the weak (or distributional) partial derivative, and $\alpha$ is a multi-index. Here $\mathscr{S}$, which is either the flow domain $\Omega$, or its boundary $\Gamma$, or part of its boundary. The norm $\|\cdot\|_{H^{m}(\mathscr{S})}$ associated with $H^{m}(\mathscr{S})$ is given by

$$
\|u\|_{H^{m}(\mathscr{S})}^{2}=\sum_{|\alpha| \leq m} \int_{\mathscr{S}}\left|D^{\alpha} u\right|^{2} d x
$$


Note that $H^{0}(\mathscr{S})=L^{2}(\mathscr{S})$ and $\|\cdot\|_{H^{0}(\mathscr{S})}=\|\cdot\|_{L^{2}(\mathscr{S})}$. For vector valued functions, we define the Sobolev space $\mathbf{H}^{m}(\mathscr{S})$ by

$$
\mathbf{H}^{m}(\mathscr{S}):=\left\{\mathbf{u}=\left(u_{1}, u_{2}\right) \mid u_{i} \in H^{m}(\mathscr{S}), \text { for } i=1,2\right\},
$$

and its associated norm

$$
\|\mathbf{u}\|_{\mathbf{H}^{m}(\mathscr{S})}^{2}=\sum_{i=1}^{2}\left\|u_{i}\right\|_{H^{m}(\mathscr{S})}^{2}
$$

\section{Existence analysis}

In this section, we establish the existence of solutions to the optimization problems. The analysis is presented based on the third test problem with homogenous Dirichlet boundary conditions. Extension to non-homogeneous Dirichlet boundary conditions can be accomplished by standard techniques. Analysis for mixed Dirichlet-Neumann boundary conditions is beyond the scope of this work. We refer the reader to [2, page 127]. Nevertheless, we note that the results given in this paper are formally valid for this case but some technical details in the analysis need to be carefully revised.

\subsection{Existence of solution to PDE constraint}

Before establishing the existence of a solution to the minimization problem, we need to first establish the existence of a solution to the the PDE constraint (the Navier-Stokes equations). To this end, the following functional spaces are introduced.

$$
\begin{array}{r}
\mathbf{H}_{\mathbf{g}}^{1}(\Omega):=\left\{\boldsymbol{\psi} \in \mathbf{H}^{1}(\Omega) \mid \boldsymbol{\psi}=\mathbf{g} \text { on } \Gamma\right\}, \\
\mathbf{H}_{0}^{1}(\Omega):=\left\{\boldsymbol{\psi} \in \mathbf{H}^{1}(\Omega) \mid \boldsymbol{\psi}=\mathbf{0} \text { on } \Gamma\right\}, \\
\mathscr{H}(\Omega):=\left\{\boldsymbol{\psi} \in \mathbf{H}_{0}^{1}(\Omega): \operatorname{div} \boldsymbol{\psi}=0 \text { in } \Omega\right\}, \\
L_{0}^{2}(\Omega)=\left\{q \in L^{2}(\Omega) \mid \int_{\Omega} q d x=0\right\} .
\end{array}
$$

Let us define the bilinear forms $a(\cdot, \cdot): \mathbf{H}^{1}(\Omega) \times \mathbf{H}^{1}(\Omega) \mapsto \mathbb{R}$ via

$$
a(\mathbf{u}, \boldsymbol{\psi})=v \int_{\Omega} \nabla \mathbf{u}: \nabla \boldsymbol{\psi} d x
$$

$b: \mathbf{H}^{1}(\Omega) \times L_{0}^{2}(\Omega) \mapsto \mathbb{R}$ via

$$
b(\mathbf{u}, q)=-\int_{\Omega} q \operatorname{div} \mathbf{u} d x,
$$

and the trilinear form $c: \mathbf{H}^{1}(\Omega) \times \mathbf{H}^{1}(\Omega) \times \mathbf{H}^{1}(\Omega) \mapsto \mathbb{R}$ via

$$
c(\mathbf{v} ; \mathbf{u}, \boldsymbol{\psi}):=\sum_{i, j=1}^{2} \int_{\Omega} v_{j} \frac{\partial u_{i}}{\partial x_{j}} \psi_{i} d x=\int_{\Omega}(\mathbf{v} \cdot \nabla) \mathbf{u} \boldsymbol{\psi} d x .
$$

The trilinear form $c$ has the following properties, [23, page 163], [8, page 285], 
1. $c(\mathbf{v} ; \boldsymbol{\psi}, \boldsymbol{\psi})=0$ for all $\mathbf{v} \in \mathscr{H}(\Omega), \boldsymbol{\psi} \in \mathbf{H}^{1}(\Omega)$.

2. $c(\mathbf{v} ; \boldsymbol{\psi}, \mathbf{u})=-c(\mathbf{v} ; \mathbf{u}, \boldsymbol{\psi})$ for all $\mathbf{v} \in \mathscr{H}(\Omega), \boldsymbol{\psi}, \mathbf{u} \in \mathbf{H}^{1}(\Omega)$.

Using our notation, the weak form of the Navier stokes equations (4-6) can be expressed as:

Given $\mathbf{f} \in \mathbf{L}^{2}(\Omega)$, find $(\mathbf{u}, p) \in \mathbf{H}_{\mathbf{g}}^{1}(\Omega) \times L_{0}^{2}(\Omega)$ such that

$$
\begin{aligned}
v a(\mathbf{u}, \boldsymbol{\psi})+c(\mathbf{u}, \mathbf{u}, \boldsymbol{\psi})+b(\boldsymbol{\psi}, p) & =(\mathbf{f}, \boldsymbol{\psi}) \text { for all } \boldsymbol{\psi} \in \mathbf{H}_{0}^{1}(\Omega), \\
b(\mathbf{u}, q) & =0 \text { for all } q \in L_{0}^{2}(\Omega) .
\end{aligned}
$$

Theorem 3.1. [23] There exists a weak solution $\mathbf{u}$ of (15) and a constant $C>0$ such that

$$
\|\mathbf{u}\|_{\mathbf{H}^{1}(\Omega)} \leq \frac{C}{v}\|\mathbf{f}\|_{\mathbf{L}^{2}(\Omega)} \text { for all } \mathbf{f} \in \mathbf{L}^{2}(\Omega)
$$

Moreover, if $\Omega$ is convex and $\Gamma$ is Lipschitz continuous, then $\mathbf{u} \in \mathbf{H}^{2}(\Omega)$.

For small values of data (f) or large enough values of $v$, uniqueness can be established:

Theorem 3.2. [23] There exists a constant $C=C(\Omega)>0$ such that the solution of (15) is unique if

$$
v^{2} \geq\left. C|| \mathbf{f}\right|_{\mathbf{L}^{2}(\Omega)} .
$$

Although the results above provide existence and uniqueness for the basic NavierStokes problems, they do not address the continuity of these solutions with respect to the parameter describing the shape of the domain. We address this question in the next subsection.

\subsection{Existence of solution to the optimization problem}

To discuss the existence of a solution to the shape optimization problem, one needs to endow the set of admissible domains $\mathscr{U}_{a d}$ with a topology and show that with respect to that topology, the set $\mathscr{U}_{a d}$ is compact. We begin with the notion of convergence of sequences of domains. We embed all domains in a fixed "hold all" domain $D$ such that $\cup_{\alpha \in \mathscr{U}_{a d}} \Omega(\alpha) \subset D$. For each $\alpha_{n} \in \mathscr{U}_{a d}$, let $\Omega_{n}=\Omega\left(\alpha_{n}\right)$. Then convergence of $\Omega_{n}$ to $\Omega$ is defined by

$$
\begin{aligned}
& \Omega_{n} \rightarrow \Omega \Longleftrightarrow \alpha_{n} \rightrightarrows \alpha \text { in }[a, b] \text { for problem } 1 \text { and } \\
& \Omega_{n} \rightarrow \Omega \Longleftrightarrow \alpha_{n} \rightrightarrows \alpha \text { in }[d, e] \text { for problem } 2,
\end{aligned}
$$

where $\alpha_{n} \rightrightarrows \alpha$ means $\alpha_{n}$ converges uniformly to $\alpha$ in a given interval. From the well known Arzelà-Ascoli theorem it follows that $\mathscr{U}_{a d}$ is compact with respect to the convergence defined in (17) [13]. Hence, to establish existence of a solution to (7), one needs to use the classical Bolzano-Weierstrass theorem for continuous functions on compact sets. 


\section{Continuity with respect to the shape}

We define the reduced cost functional $\hat{J}_{i}: \mathscr{U}_{a d} \mapsto \mathbb{R}, i=1,2,3$ by $\hat{J}_{i}(\Omega)=J_{i}(\mathbf{u}(\Omega), \Omega)$. In order to obtain the existence of optimal shapes we need the continuity, or at least the lower semi-continuity of the shape functional $\hat{J}_{i}(\Omega)$. Since our shape functionals depends on the solution $\mathbf{u}(\Omega)$ of a partial differential equation, we need the continuity of this solution with respect to the shape for an appropriate topology of domains, in particular the topology defined in (17). The following lemma will become important in what follows

Lemma 3.1. [13, page 26] Let $\Omega\left(\alpha_{n}\right) \rightarrow \Omega(\alpha), n \rightarrow \infty$, and let $\chi_{n}$, $\chi$ be the characteristic functions of $\Omega\left(\alpha_{n}\right), \Omega(\alpha)$, respectively. Then

$$
\chi_{n} \rightarrow \chi \text { in } L^{p}(D), \text { for all } p \in[1, \infty) .
$$

We begin with the establishment of the continuity of $\mathbf{u}(\Omega)$ with respect to the shape of the domain $\Omega$. The domains $\Omega(\alpha)$ are uniformly Lipschitz continuous for each $\alpha \in \mathscr{U}_{a d}$, and hence, have the uniform extension property, [5]. Let $D$ be the hold all domain as in the above Lemma and define the extension operator

$$
\varepsilon_{\Omega}: H_{0}^{1}(\Omega) \mapsto H_{0}^{1}(D)
$$

by

$$
\left(\varepsilon_{\Omega} u\right)(x)=\left\{\begin{array}{cc}
u(x), & x \in \Omega \\
0, & x \in D \backslash \Omega .
\end{array}\right.
$$

We shall use the symbol $\tilde{u}$ to denote the extension of $u$ from $H_{0}^{1}(\Omega)$ to $H_{0}^{1}(D)$.

Definition 3.1. Let $\mathbf{u}_{n} \in \mathbf{H}_{0}^{1}\left(\Omega_{n}\right), \mathbf{u} \in \mathbf{H}_{0}^{1}(\Omega), \alpha_{n}, \alpha \in \mathscr{U}_{a d}$. We say that

$$
\begin{array}{r}
\mathbf{u}_{n} \rightarrow \mathbf{u} \text { if and only if } \tilde{\mathbf{u}}_{n} \rightarrow \tilde{\mathbf{u}} \text { in } \mathbf{H}_{0}^{1}(D), \\
\mathbf{u}_{n} \rightarrow \mathbf{u} \text { if and only if } \tilde{\mathbf{u}}_{n} \rightarrow \tilde{\mathbf{u}} \text { in } \mathbf{H}_{0}^{1}(D), n \rightarrow \infty,
\end{array}
$$

where the symbols $\rightarrow$ and $\rightarrow$ in (18) denote strong and weak convergence in $\mathbf{H}_{0}^{1}(\Omega)$ respectively.

Lemma 3.2. Let $\alpha_{n}, \alpha \in \mathscr{U}_{a d}$, be such that $\Omega\left(\alpha_{n}\right) \rightarrow \Omega(\alpha), n \rightarrow \infty$ and let $\mathbf{u}_{n}:=\mathbf{u}\left(\alpha_{n}\right)$ be the solution to the Navier-Stokes equations in $\Omega_{n}$. Then

$$
\tilde{\mathbf{u}}_{n} \rightarrow \tilde{\mathbf{u}} \text { in } \mathbf{H}_{0}^{1}(D), n \rightarrow \infty .
$$

Proof. By Theorem 3.1, we have

$$
\left\|\tilde{\mathbf{u}}_{n}\right\|_{\mathbf{H}^{1}(D)}=\left\|\mathbf{u}_{n}\right\|_{\mathbf{H}^{1}\left(\Omega_{n}\right)} \leq \frac{C}{v}\|\mathbf{f}\|_{\mathbf{L}^{2}(D)},
$$

with a constant $C$ independent of $n$. From this, one can pass to a subsequence $\left\{\tilde{\mathbf{u}}_{n_{k}}\right\}$ of $\left\{\tilde{\mathbf{u}}_{n}\right\}$ such that

$$
\tilde{\mathbf{u}}_{n_{k}} \rightarrow \tilde{\mathbf{u}} \text { in } \mathbf{H}_{0}^{1}(D), \quad k \rightarrow \infty .
$$


We first prove that $\left.\tilde{\mathbf{u}}\right|_{D \backslash \bar{\Omega}}=0$ which implies that $\left.\tilde{\mathbf{u}}\right|_{\Omega} \in \mathbf{H}_{0}^{1}(\Omega)$. Since $\mathbf{H}_{0}^{1}(D)$ embeds compactly into $\mathbf{L}^{2}(D)$,

$$
\tilde{\mathbf{u}}_{n_{k}} \rightarrow \tilde{\mathbf{u}} \text { in } \mathbf{L}^{2}(D), \quad k \rightarrow \infty .
$$

If $\chi_{\bar{\Omega}^{C}}$ denotes the characteristic function of the complement of $\bar{\Omega}$, then Theorem 4.3 in [7, page 176] and Fatou's Lemma imply

$$
\begin{aligned}
\int_{D} \chi_{\bar{\Omega}^{c}} \tilde{\mathbf{u}}^{2} d x & \leq \int_{D} \liminf _{k \rightarrow \infty} \chi_{\bar{\Omega}_{n_{k}}} \tilde{\mathbf{u}}^{2} d x \leq \liminf _{k \rightarrow \infty} \int_{D} \chi_{\bar{\Omega}_{n_{k}}^{C}} \tilde{\mathbf{u}}^{2} d x \\
& =\liminf _{k \rightarrow \infty} \int_{D} \chi_{\bar{\Omega}_{n_{k}}^{C}}\left|\tilde{\mathbf{u}}-\tilde{\mathbf{u}}_{n_{k}}\right|^{2} d x \leq \lim _{k \rightarrow \infty} \int_{D}\left|\tilde{\mathbf{u}}-\tilde{\mathbf{u}}_{n_{k}}\right|^{2} d x=0 .
\end{aligned}
$$

As a consequence, $\tilde{\mathbf{u}}=0$ holds almost everywhere on $\bar{\Omega}^{C}$ which implies that

$$
\mathbf{u}_{\Omega}=\left.\tilde{\mathbf{u}}\right|_{\Omega} \in \mathbf{H}_{0}^{1}(\Omega)
$$

We need to show that $\left.\tilde{\mathbf{u}}\right|_{\Omega}$ solves

$$
v \int_{\Omega} \nabla \mathbf{u}: \nabla \boldsymbol{\psi} d x+\int_{\Omega}(\mathbf{u} \cdot \nabla) \mathbf{u} \boldsymbol{\psi} d x=\int_{\Omega} \mathbf{f} \boldsymbol{\psi} d x, \text { for all } \boldsymbol{\psi} \in \mathscr{H}(\Omega) .
$$

Observe that $\tilde{\mathbf{u}}_{n}$ satisfies

$$
v \int_{D} \chi_{n} \nabla \tilde{\mathbf{u}}_{n}: \nabla \tilde{\boldsymbol{\psi}} d x+\int_{D} \chi_{n}\left(\tilde{\mathbf{u}}_{n} \cdot \nabla\right) \tilde{\mathbf{u}}_{n} \tilde{\boldsymbol{\psi}} d x=\int_{D} \chi_{n} \mathbf{f} \tilde{\boldsymbol{\psi}} d x, \quad \tilde{\boldsymbol{\psi}} \in \mathscr{H}\left(\Omega_{n}\right),
$$

where $\chi_{n}$ is the characteristic function of $\Omega_{n}$. Choose

$$
\boldsymbol{\xi} \in \mathbf{V}(\Omega)=\left\{\boldsymbol{\phi} \in\left(C_{0}^{\infty}(\Omega)\right)^{2} \mid \operatorname{div} \boldsymbol{\phi}=0 \text { in } \Omega\right\} .
$$

Then there exists $N_{\boldsymbol{\xi}} \in \mathbb{N}$ such that $\operatorname{supp}(\boldsymbol{\xi}) \subset \Omega_{n}$ for $n \geq N_{\boldsymbol{\xi}}$ [7, page 264]. Consequently, we may use $\tilde{\boldsymbol{\xi}}$ as a test function in (23):

$$
v \int_{D} \chi_{n_{k}} \nabla \tilde{\mathbf{u}}_{n_{k}}: \nabla \tilde{\boldsymbol{\xi}} d x+\int_{D} \chi_{n_{k}}\left(\tilde{\mathbf{u}}_{n_{k}} \cdot \nabla\right) \tilde{\mathbf{u}}_{n_{k}} \tilde{\boldsymbol{\xi}} d x=\int_{D} \chi_{n_{k}} \mathbf{f} \tilde{\xi} d x .
$$

Moreover, the extension $\tilde{\xi}$ belongs to

$$
\mathbf{V}(D)=\left\{\boldsymbol{\phi} \in\left(C_{0}^{\infty}(D)\right)^{2} \mid \operatorname{div} \boldsymbol{\phi}=0 \text { in } D\right\} .
$$

We examine each term in (24) separately. We first note that

$$
\begin{gathered}
v \int_{D} \chi_{n_{k}} \nabla \tilde{\mathbf{u}}_{n_{k}}: \nabla \tilde{\boldsymbol{\xi}} d x \stackrel{n \rightarrow \infty}{\longrightarrow} v \int_{D} \chi \nabla \tilde{\mathbf{u}}: \nabla \tilde{\boldsymbol{\xi}} d x \text { (by equation (20)), } \\
=v \int_{\Omega} \nabla \mathbf{u}: \nabla \boldsymbol{\xi} d x .
\end{gathered}
$$

Next, we estimate the nonlinear term. Integrating by parts, one obtains

$$
\begin{gathered}
\left.\int_{D} \chi_{n_{k}}\left(\tilde{\mathbf{u}}_{n_{k}} \cdot \nabla\right) \tilde{\mathbf{u}}_{n_{k}}, \tilde{\boldsymbol{\xi}}\right) d x=\int_{\partial D} \chi_{n_{k}}\left(\tilde{\mathbf{u}}_{n_{k}} \cdot \tilde{\boldsymbol{\xi}}\right)\left(\tilde{\mathbf{u}}_{n_{k}} \cdot \mathbf{n}\right) d s \\
-\int_{D} \chi_{n_{k}} \tilde{\mathbf{u}}_{n_{k}} \cdot \tilde{\boldsymbol{\xi}} \operatorname{div} \tilde{\mathbf{u}}_{n_{k}} d x-\int_{D} \chi_{n_{k}} \tilde{\mathbf{u}}_{n_{k}} \cdot\left(\tilde{\mathbf{u}}_{n_{k}} \cdot \nabla\right) \tilde{\boldsymbol{\xi}} d x
\end{gathered}
$$


We have $\int_{\partial D} \chi_{n_{k}}\left(\tilde{\mathbf{u}}_{n_{k}} \cdot \tilde{\boldsymbol{\xi}}\right)\left(\tilde{\mathbf{u}}_{n_{k}} \cdot \mathbf{n}\right) d s=0$, since $\left.\tilde{\mathbf{u}}_{n_{k}}\right|_{\Gamma}=0$ for every $n$. Further note that $\boldsymbol{\xi}_{i}$ and $(\nabla \boldsymbol{\xi})_{i, j}$ belong to $L^{\infty}(\Omega)$. Since $\left\|\operatorname{div} \tilde{\mathbf{u}}_{n_{k}}\right\|_{L^{2}(D)} \leq\left\|\tilde{\mathbf{u}}_{n_{k}}\right\|_{\mathbf{H}^{1}(D)}<\infty$ for all $n$, we may extract a subsequence, again denoted by $\tilde{\mathbf{u}}_{n_{k}}$ such that

$$
\operatorname{div} \tilde{\mathbf{u}}_{n_{k}} \rightarrow \operatorname{div} \tilde{\mathbf{u}} \text { in } L^{2}(D)
$$

Hence, using Lemma 3.1, equations (20) and (26) lead to

$$
\begin{aligned}
\int_{D} \chi_{n_{k}} \tilde{\mathbf{u}}_{n_{k}} \cdot \tilde{\boldsymbol{\xi}} \operatorname{div} \tilde{\mathbf{u}}_{n_{k}} d x-\int_{D} \chi_{n_{k}} \tilde{\mathbf{u}} \cdot \tilde{\boldsymbol{\xi}} \operatorname{div} \tilde{\mathbf{u}} d x=\int_{D} \chi_{n_{k}} \operatorname{div} \tilde{\mathbf{u}}_{n_{k}}\left(\tilde{\mathbf{u}}_{n_{k}}-\tilde{\mathbf{u}}\right) \tilde{\boldsymbol{\xi}} d x \\
\quad+\int_{D} \chi_{n_{k}}\left(\operatorname{div} \tilde{\mathbf{u}}_{n_{k}}-\operatorname{div} \tilde{\mathbf{u}}\right) \tilde{\mathbf{u}} \cdot \tilde{\boldsymbol{\xi}} d x+\int_{D}\left(\chi_{n_{k}}-\chi\right) \operatorname{div} \tilde{\mathbf{u}} \tilde{\mathbf{u}} \cdot \tilde{\boldsymbol{\xi}} d x \stackrel{n \rightarrow \infty}{\longrightarrow} 0
\end{aligned}
$$

Thus

$$
\int_{D} \chi_{n_{k}} \tilde{\mathbf{u}}_{n_{k}} \cdot \tilde{\boldsymbol{\xi}} \operatorname{div} \tilde{\mathbf{u}}_{n_{k}} d x \stackrel{n \rightarrow \infty}{\longrightarrow} \int_{D} \chi \tilde{\mathbf{u}} \cdot \tilde{\boldsymbol{\xi}} \operatorname{div} \tilde{\mathbf{u}} d x=\int_{\Omega} \mathbf{u} \cdot \boldsymbol{\xi} \operatorname{div} \mathbf{u} d x .
$$

In a similar fashion, we have

$$
\int_{D} \chi_{n_{k}} \tilde{\mathbf{u}}_{n_{k}} \cdot\left(\tilde{\mathbf{u}}_{n_{k}} \cdot \nabla\right) \tilde{\boldsymbol{\xi}} d x \stackrel{n \rightarrow \infty}{\longrightarrow} \int_{D} \chi \tilde{\mathbf{u}} \cdot(\tilde{\mathbf{u}} \cdot \nabla) \tilde{\boldsymbol{\xi}} d x=\int_{\Omega} \mathbf{u} \cdot(\mathbf{u} \cdot \nabla) \boldsymbol{\xi} d x
$$

Therefore, from (A)

$$
\int_{D} \chi_{n_{k}}\left(\tilde{\mathbf{u}}_{n_{k}} \cdot \nabla\right) \tilde{\mathbf{u}}_{n_{k}} \tilde{\boldsymbol{\xi}} d x \stackrel{n \rightarrow \infty}{\longrightarrow} \int_{D} \chi(\tilde{\mathbf{u}} \cdot \nabla) \tilde{\mathbf{u}} \tilde{\xi} d x=\int_{\Omega}(\mathbf{u} \cdot \nabla) \mathbf{u} \xi d x .
$$

Finally,

$$
\int_{D} \chi_{n_{k}} \mathbf{f} \tilde{\boldsymbol{\psi}} d x-\int_{D} \chi \mathbf{f} \tilde{\boldsymbol{\psi}} d x=\int_{D}\left(\chi_{n_{k}}-\chi\right) \mathbf{f} \tilde{\boldsymbol{\psi}} d x \stackrel{n \rightarrow \infty}{\longrightarrow} 0 .
$$

Since $V(\Omega)$ is dense in $\mathscr{H}(\Omega)$ (see [8, page 26]), we conclude that $\left.\tilde{\mathbf{u}}\right|_{\Omega}$ solves (22) and moreover $\left.\tilde{\mathbf{u}}\right|_{\Omega}$ is divergent free in $\Omega$ [13, page 85$]$. The function $\left.\tilde{\mathbf{u}}\right|_{\Omega}$ is the unique solution to (15) so that not only a subsequence but the whole sequence tends weakly to $\tilde{\mathbf{u}}$ in $\mathbf{H}_{0}^{1}(D)$. To prove strong convergence, from the definition of the problem on $\Omega_{n}$ and properties of $c$ over $\mathscr{H}(\Omega)$ in Lemma 3.1, it follows that

$$
v \int_{D} \chi_{n}\left|\nabla \tilde{\mathbf{u}}_{n}\right|^{2} d x=\int_{D} \chi_{n} \mathbf{f} \tilde{\mathbf{u}}_{n} d x \rightarrow \int_{D} \chi \mathbf{f} \tilde{\mathbf{u}} d x=v \int_{D} \chi|\nabla \tilde{\mathbf{u}}|^{2} d x
$$

The next task is to prove the continuity of $\hat{J}_{i}(\Omega)$. We need to show that if the domains converge in the sense of (17), then $\hat{J}_{i}\left(\Omega_{n}\right) \mapsto \hat{J_{i}}(\Omega), n \rightarrow \infty$. This is established for cost functionals $\hat{J}_{1}(\Omega)$ and $\hat{J}_{2}(\Omega)$ in [21]. Therefore, we only discuss continuity of $\hat{J}_{3}(\Omega)$ in the following lemma.

Lemma 3.3. Let $J_{3}\left(\mathbf{u}_{n}, \Omega_{n}\right)=\int_{\Omega_{n}} g_{3}\left(\operatorname{det} \nabla \boldsymbol{u}_{n}\right) d x$. Then the cost functional $\hat{J}_{3}(\Omega)$ is continuous on $\mathscr{U}_{\text {ad }}$. 
Proof. Let us first note that $t^{3} /\left(t^{2}+1\right) \leq t$, hence, it follows that for $\mathbf{u} \in \mathbf{H}_{0}^{1}(\Omega)$,

$$
\int_{\Omega} g_{3}(\operatorname{det} \nabla \mathbf{u}) d x \leq \int_{\Omega}|\operatorname{det} \nabla \mathbf{u}| d x .
$$

Also note that by Young's inequality

$$
\begin{aligned}
|\operatorname{det} \nabla \mathbf{u}| & =\left|\frac{\partial u_{1}}{\partial x_{1}} \frac{\partial u_{2}}{\partial x_{2}}-\frac{\partial u_{2}}{\partial x_{1}} \frac{\partial u_{1}}{\partial x_{2}}\right| \\
& \leq \frac{1}{2}\left[\left(\frac{\partial u_{1}}{\partial x_{1}}\right)^{2}+\left(\frac{\partial u_{2}}{\partial x_{2}}\right)^{2}+\left(\frac{\partial u_{2}}{\partial x_{1}}\right)^{2}+\left(\frac{\partial u_{1}}{\partial x_{2}}\right)^{2}\right] \\
& =\frac{1}{2}(\nabla \mathbf{u}: \nabla \mathbf{u})
\end{aligned}
$$

Hence, for $\mathbf{u} \in \mathbf{H}_{0}^{1}(\Omega)$,

$$
\int_{\Omega} g_{3}(\operatorname{det} \nabla \mathbf{u}) d x \leq \frac{1}{2}\|\nabla \mathbf{u}\|_{\mathbf{L}^{2}(\Omega)}^{2} \leq \frac{1}{2}\|\mathbf{u}\|_{\mathbf{H}^{1}(\Omega)}^{2} .
$$

The function $g_{3}(t)$ is globally Lipschitz with constant $3 / 2$, i.e.,

$$
\left|g_{3}(t)-g_{3}(s)\right| \leq \frac{3}{2}|t-s|, \quad 0 \leq t, s \in \mathbb{R} .
$$

Consequently, we estimate

$$
\begin{aligned}
\left|J_{3}\left(\mathbf{u}_{n}, \Omega_{n}\right)-J_{3}(\mathbf{u}, \Omega)\right| & =\left|\int_{\Omega_{n}} g_{3}\left(\operatorname{det} \nabla \mathbf{u}_{n}\right) d x-\int_{\Omega} g_{3}(\operatorname{det} \nabla \mathbf{u}) d x\right|, \\
& \leq \int_{D}\left|g_{3}\left(\operatorname{det} \nabla \tilde{\mathbf{u}}_{n}\right)-g_{3}(\operatorname{det} \nabla \tilde{\mathbf{u}})\right| d x
\end{aligned}
$$

Using (29) with $t=\operatorname{det} \nabla \tilde{\mathbf{u}}_{n}, s=\operatorname{det} \nabla \tilde{\mathbf{u}}$, we find

$$
\int_{D}\left|g_{3}\left(\operatorname{det} \nabla \tilde{\mathbf{u}}_{n}\right)-g_{3}(\operatorname{det} \nabla \tilde{\mathbf{u}})\right| d x \leq \frac{3}{2} \int_{D}\left|\operatorname{det} \nabla \tilde{\mathbf{u}}_{n}-\operatorname{det} \nabla \tilde{\mathbf{u}}\right| d x .
$$

Hence,

$$
\begin{aligned}
& \left|J_{3}\left(\mathbf{u}_{n}, \Omega_{n}\right)-J_{3}(\mathbf{u}, \Omega)\right| \leq \frac{3}{2} \int_{D}\left|\operatorname{det} \nabla \tilde{\mathbf{u}}_{n}-\operatorname{det} \nabla \tilde{\mathbf{u}}\right| d x, \\
& \leq \frac{3}{2} \int_{D}|\underbrace{\frac{\partial \tilde{u}_{1}^{n}}{\partial x_{1}} \frac{\partial \tilde{u}_{2}^{n}}{\partial x_{2}}-\frac{\partial \tilde{u}_{1}}{\partial x_{1}} \frac{\partial \tilde{u}_{2}}{\partial x_{2}}}_{A}|+|\underbrace{\frac{\partial \tilde{u}_{2}^{n}}{\partial x_{1}} \frac{\partial \tilde{u}_{1}^{n}}{\partial x_{2}^{n}}-\frac{\partial \tilde{u}_{2}}{\partial x_{1}} \frac{\partial \tilde{u}_{1}}{\partial x_{2}}}_{B}| d x .
\end{aligned}
$$

Note that by using the relation $\left|a^{n} b^{n}-a b\right| \leq\left|a^{n}\left(b^{n}-b\right)\right|+\left|\left(a^{n}-a\right) b\right|$, and using the Cauchy-Schwarz inequality, we have that

$$
\int_{\Omega}\left|a^{n} b^{n}-a b\right| d x \leq\left\|\left.a^{n}\right|_{L^{2}}|| b^{n}-b||_{L^{2}}+\right\| b^{n}||_{L^{2}}|| a^{n}-\left.a\right|_{L^{2}} .
$$


With $a=\frac{\partial \tilde{u}_{1}}{\partial x_{1}}, b=\frac{\partial \tilde{u}_{2}}{\partial x_{2}}$, we find

$$
\int_{D}|A| d x \leq\left.\left\|\frac{\partial \tilde{u}_{1}^{n}}{\partial x_{1}}\right\|\right|_{L^{2}(D)}\left\|\frac{\partial \tilde{u}_{2}^{n}}{\partial x_{2}}-\frac{\partial \tilde{u}_{2}}{\partial x_{2}}\right\|_{L^{2}(D)}+\left\|\frac{\partial \tilde{u}_{2}^{n}}{\partial x_{2}}\right\|_{L^{2}(D)}\left\|\frac{\partial \tilde{u}_{1}^{n}}{\partial x_{1}}-\frac{\partial \tilde{u}_{1}}{\partial x_{1}}\right\|_{L^{2}(D)}
$$

and similarly for $\int_{D}|B| d x$. Let $\Omega_{n} \rightarrow \Omega$, as $n \rightarrow \infty$. Then from Lemma 3.2, it follows that $\tilde{\mathbf{u}}_{n} \rightarrow \tilde{\mathbf{u}}$ in $\mathbf{H}_{0}^{1}(D)$. Since by (28), the terms $\left\|\frac{\partial \tilde{u}_{1}^{n}}{\partial x_{1}}\right\|_{L^{2}(D)},\left\|\frac{\partial \tilde{u}_{2}^{n}}{\partial x_{2}}\right\|_{L^{2}(D)},\left.\left\|\frac{\partial \tilde{u}_{2}^{n}}{\partial x_{1}}\right\|\right|_{L^{2}(D)}$, and $\left\|\frac{\partial \tilde{u}_{1}^{n}}{\partial x_{2}}\right\|_{L^{2}(D)}$ are uniformly bounded with respect to $n$, it follows that $J_{3}\left(\mathbf{u}_{n}, \Omega_{n}\right) \rightarrow$ $J_{3}(\mathbf{u}, \Omega)$.

Lemma 3.4. The optimization problem (7) has a solution.

Proof. Since $J_{1}, J_{2}$ and $J_{3}$ are continuous and $\mathscr{U}_{a d}$ is compact, the Bolzano-Weierstrass theorem asserts that $J_{1}, J_{2}$ and $J_{3}$ attain global minima on $\mathscr{U}_{a d}$.

\section{Sensitivity analysis}

In this section, we discuss the necessary optimality conditions for (7). In order to set up the optimality system, a common technique is to introduce a family of perturbations $\Omega_{t}$ of a given admissible domain $\Omega$ which depend on a parameter $t$. The relationship of these perturbations to the set of admissible domains that was considered in Section 2 , is given in Remark 4.1 below. This family of perturbations of $\Omega$ can be constructed for instance by perturbation of the identity, see, e.g., [22],[7],[13]. Let $\Omega \subset \bar{D}$ be open and let

$$
\mathscr{S}_{d}=\left\{\mathbf{h} \in C^{1,1}(\bar{D}):\left.\mathbf{h}\right|_{\partial D}=0\right\}
$$

be the space of deformation fields which define for $t>0$ a perturbation of $\Omega$ by

$$
\begin{aligned}
T_{t}: D & \mapsto \mathbb{R}^{2}, \\
x & \mapsto T_{t}(x)=x+t \mathbf{h}(x) .
\end{aligned}
$$

Then for each $\mathbf{h} \in \mathscr{S}_{d}$, there exists $\tau>0$ such that $T_{t}(D)=D$ and $\left\{T_{t}\right\}$ is a family of $C^{1,1}$-diffeomorphisms for $|t|<\tau$. For each $t \in \mathbb{R}$ with $|t|<\tau$, we set

$$
\Omega_{t}=T_{t}(\Omega), \Gamma_{t}=T_{t}(\Gamma) .
$$

Thus $\Omega_{0}=\Omega, \Gamma_{0}=\Gamma, \Omega_{t} \subset D$.

Remark 4.1. The form of the deformation field $\mathbf{h}$ realizing shape variations depends on how shapes of admissible domains are parametrized [13]. Therefore, for the family of domains shown in Figures $2(a)$ and $2(b)$, it is natural to take the deformation fields of the form $\mathbf{h}=\left(0, h_{2}\right)$ and $\mathbf{h}=\left(h_{1}, 0\right)$, respectively. Here $h_{i}$ corresponds to the variation $\delta \alpha=\tilde{\alpha}-\alpha$, where $\tilde{\alpha} \in \mathscr{U}_{\text {ad }}$ is a function that determines $\Gamma_{f}(\tilde{\alpha})$ after deformation of $\Gamma_{f}(\alpha)$ by $\delta \alpha$. Moreover, $\delta \alpha$ is defined on the boundary $\Gamma_{f}$. Therefore, to avoid ambiguity with (33)-(34), we must extend this variation such that it is defined over the entire domain. We shall discuss this later in Section 5. 
The Eulerian derivative of $J$ at $\Omega$ in the direction of the deformation field $\mathbf{h}$ is defined as

$$
d J(\mathbf{u}, \Omega) \mathbf{h}=\lim _{t \rightarrow 0} \frac{J\left(\mathbf{u}_{t}, \Omega_{t}\right)-J(\mathbf{u}, \Omega)}{t} .
$$

The functional $J$ is called shape differentiable at $\Omega$ if $d J(\mathbf{u}, \Omega) \mathbf{h}$ exists for all $\mathbf{h} \in \mathscr{S}_{d}$ and $\mathbf{h} \mapsto d J(\mathbf{u}, \Omega) \mathbf{h}$ defines a continuous linear functional on $\mathscr{S}_{d}$. To compute (35), we compute the derivative of the state equation first, using the classical results of shape calculus as in [4],[22]. The shape derivative of the state variables $(\mathbf{u}, p)$ is the solution of the following linear systems [14],[4]:

$$
\left\{\begin{array}{l}
-v \Delta \mathbf{u}^{\prime}+D \mathbf{u} \cdot \mathbf{u}^{\prime}+D \mathbf{u}^{\prime} \cdot \mathbf{u}+\nabla p^{\prime}=0 \text { in } \Omega, \\
\operatorname{div} \mathbf{u}^{\prime}=0 \text { in } \Omega,
\end{array}\right.
$$

supplemented with the following boundary conditions for test cases 1 and 2

$$
\left\{\begin{array}{l}
\mathbf{u}^{\prime}=\mathbf{0} \text { on } \Gamma_{\text {in }} \cup \Gamma_{w}, \\
\mathbf{u}^{\prime}=-\frac{\partial(\mathbf{u}-\mathbf{g})}{\partial \mathbf{n}} \mathbf{h} \cdot \mathbf{n} \text { on } \Gamma_{f}, \\
-p^{\prime} \mathbf{n}+v \frac{\partial \mathbf{u}^{\prime}}{\partial \mathbf{n}}=0 \text { on } \Gamma_{\text {out }},
\end{array}\right.
$$

and

$$
\left\{\begin{array}{l}
\mathbf{u}^{\prime}=\mathbf{0} \text { on } \Gamma \backslash \Gamma_{f}, \\
\mathbf{u}^{\prime}=-\frac{\partial(\mathbf{u}-\mathbf{g})}{\partial \mathbf{n}} \mathbf{h} \cdot \mathbf{n} \text { on } \Gamma_{f},
\end{array}\right.
$$

for case 3. In what follows, we will need the following two lemmas.

Lemma 4.1. (Transport theorem) $[22]$ Let $f \in C\left([0, \tau], W^{1,1}(D)\right)$, ( $\tau$ sufficiently small), and assume that $f_{t}(0)$ exists in $L^{1}(D)$. Then

$$
\left.\frac{d}{d t} \int_{\Omega_{t}} f(t, x) d x\right|_{t=0}=\int_{\Omega} f_{t}(0, x) d x+\int_{\Gamma} f(0, x) \mathbf{h} \cdot \mathbf{n} d s .
$$

Lemma 4.2. The shape derivative $\mathbf{u}^{\prime}$ in (36) satisfies

$$
\mathbf{u}^{\prime} \cdot \mathbf{n}=0 \text {, on } \Gamma_{f} .
$$

Proof. Since $\mathbf{u}^{\prime}=-(D(\mathbf{u}-\mathbf{g}) \cdot \mathbf{n}) \mathbf{h} \cdot \mathbf{n}$ on $\Gamma_{f}$, by using the tangential divergence formula [22, Page 82], we have that:

$$
\mathbf{u}^{\prime} \cdot \mathbf{n}=(D(\mathbf{u}-\mathbf{g}) \cdot \mathbf{n}) \cdot \mathbf{n}(\mathbf{h} \cdot \mathbf{n})=\left.\operatorname{div}(\mathbf{u}-\hat{\mathbf{g}})(\mathbf{h} \cdot \mathbf{n})\right|_{\Gamma_{f}}-\operatorname{div}_{\Gamma_{f}}(\mathbf{u}-\mathbf{g})(\mathbf{h} \cdot \mathbf{n}),
$$

where $\hat{\mathbf{g}}$ is any $C^{1}$ divergence free extension of $\mathbf{g}$ to an open neighbourhood of $\Gamma \subset \mathbb{R}^{2}$. Using $\mathbf{u}-\mathbf{g}=0$ on $\Gamma_{f}$, as well as considering the fact that we are using divergence free fields, the expression on the right-hand side in (40) vanishes and this completes the proof. 


\subsection{Shape gradients of cost functionals}

This subsection is devoted to the computation of the Eulerian derivatives of $J_{i}(\Omega, \mathbf{u}), i=$ $1, \ldots, 3$. The goal is to express (35) in the Hardamard-Zolesio structure form $d J_{i}(\Omega, \mathbf{u}) \mathbf{h}=$ $\int_{\Gamma_{f}} \nabla J_{i} \mathbf{n} \cdot \mathbf{h}$, under appropriate smoothness conditions on the boundary of $\Omega$. We call $\nabla J_{i} \mathbf{n}$, the shape gradient of $J_{i}$. This gradient depends only on the state variables $(\mathbf{u}, p)$ and the adjoint state variables $(\boldsymbol{\lambda}, q)$. The variables $(\boldsymbol{\lambda}, q)$ are defined by

$$
\left\{\begin{array}{l}
-v \Delta \boldsymbol{\lambda}-D \boldsymbol{\lambda} \cdot \mathbf{u}+(D \mathbf{u})^{t} \cdot \boldsymbol{\lambda}+\nabla q=J_{i}(\mathbf{u})^{\prime} \text { in } \Omega \\
\operatorname{div} \boldsymbol{\lambda}=0 \text { in } \Omega
\end{array}\right.
$$

together with the boundary conditions

$$
\left\{\begin{array}{l}
\lambda=0 \text { on } \Gamma_{w} \cup \Gamma_{f} \cup \Gamma_{\text {in }}, \\
q \mathbf{n}-v D \lambda \cdot \mathbf{n}-(\mathbf{u} \cdot \mathbf{n}) \boldsymbol{\lambda}=0 \text { on } \Gamma_{\text {out }},
\end{array}\right.
$$

for test cases 1 and 2 and

$$
\lambda=0 \text { on } \Gamma,
$$

for test case 3 . In what follows, we will for simplicity, consider system $(41,43)$ in our computations. Nevertherless, the results still remain valid if we use system $(41,42)$.

Theorem 4.1. Let $\Omega \subset \mathbb{R}^{2}$ be a bounded, open and connected domain, with a piecewise smooth boundary of class $C^{2}$ and convex corners, (see, e.g., [23],[9]), $\mathbf{u}_{d}$ the desired flow field and $\mathbf{h}$ a fixed vector field. Then the shape gradients $\nabla J_{i} \mathbf{n}$ of the 3 cost functionals $J_{1}, J_{2}$ and $J_{3}$ can be expressed as

$$
\begin{aligned}
& \nabla J_{1} \mathbf{n}=\left[\frac{1}{2}\left|\mathbf{u}-\mathbf{u}_{d}\right|^{2}+v(D(\mathbf{u}-\mathbf{g}) \cdot \mathbf{n}) \cdot(D \boldsymbol{\lambda} \cdot \mathbf{n})\right] \mathbf{n}, \\
& \nabla J_{2} \mathbf{n}=\left[\frac{1}{2}|\operatorname{curl} \mathbf{u}|^{2}+D(\mathbf{u}-\mathbf{g}) \cdot \mathbf{n} \cdot(v D \boldsymbol{\lambda} \cdot \mathbf{n}-(\operatorname{curl} \mathbf{u}) \boldsymbol{\tau})\right] \mathbf{n}, \\
& \nabla J_{3} \mathbf{n}=\left[g_{3}(\operatorname{det} \nabla \mathbf{u})+(D(\mathbf{u}-\mathbf{g}) \cdot \mathbf{n}) \cdot(v D \boldsymbol{\lambda} \cdot \mathbf{n}-P(\mathbf{u}))\right] \mathbf{n},
\end{aligned}
$$

where all expressions are evaluated on $\Gamma_{f}$, and the adjoint state $\lambda$ satisfies $(41,43)$ with $J_{1}^{\prime}(\mathbf{u})=\left(\mathbf{u}-\mathbf{u}_{d}\right), J_{2}^{\prime}(\mathbf{u})=\operatorname{curl}($ curl $\mathbf{u})=-\Delta \mathbf{u}$, and $J_{3}^{\prime}(\mathbf{u})=R(\mathbf{u})$, where

$$
R(\mathbf{u})=\left(\begin{array}{c}
-\operatorname{curl}\left(g_{3}^{\prime}(\operatorname{det} \nabla \mathbf{u}) \nabla u_{2}\right) \\
\operatorname{curl}\left(g_{3}^{\prime}(\operatorname{det} \nabla \mathbf{u}) \nabla u_{1}\right)
\end{array}\right),
$$

and

$$
P(\mathbf{u})=\left(\begin{array}{c}
g_{3}^{\prime}(\operatorname{det} \nabla \mathbf{u})\left(\frac{\partial u_{2}}{\partial x_{2}} n_{x_{1}}-\frac{\partial u_{2}}{\partial x_{1}} n_{x_{2}}\right) \\
g_{3}^{\prime}(\operatorname{det} \nabla \mathbf{u})\left(\frac{\partial u_{1}}{\partial x_{1}} n_{x_{2}}-\frac{\partial u_{1}}{\partial x_{2}} n_{x_{1}}\right)
\end{array}\right)
$$


Proof. The proofs of expressions (44-45) can be found in, e.g., [21]. Therefore, we shall only prove expression (46). Since $J_{3}(\Omega)$ is differentiable with respect to $\mathbf{u}$, and the state $\mathbf{u}$ is differentiable with respect to $t$, using Lemma 4.1 we obtain the Eulerian derivative of $J_{3}(\Omega)$ with respect to $t$ :

$$
\begin{aligned}
d J_{3}(\Omega ; \mathbf{u}) \mathbf{h}= & \int_{\Omega} g_{3}^{\prime}\left(u_{x_{1}}^{1}\left(u_{2}^{\prime}\right)_{x_{2}}+\left(u_{1}^{\prime}\right)_{x_{1}} u_{x_{2}}^{2}-u_{x_{1}}^{2}\left(u_{1}^{\prime}\right)_{x_{2}}-u_{x_{2}}^{1}\left(u_{2}^{\prime}\right)_{x_{1}}\right) d x \\
& +\int_{\Gamma} g_{3}(\operatorname{det} \nabla \mathbf{u}) \mathbf{h} \cdot \mathbf{n} d s,
\end{aligned}
$$

where $u_{1}^{\prime}:=\frac{\partial u^{1}}{\partial t}, u_{2}^{\prime}:=\frac{\partial u^{2}}{\partial t}, \mathbf{u}^{\prime}=\left(u_{1}^{\prime}, u_{2}^{\prime}\right)$, and $g_{3}^{\prime} \equiv g_{3}^{\prime}(\operatorname{det} \nabla \mathbf{u})$.

Using integration by parts, the first term on the right hand side of (48) can be written as

$$
\begin{aligned}
\int_{\Omega} g_{3}^{\prime}\left(u_{x_{1}}^{1}\left(u_{2}^{\prime}\right)_{x_{2}}+\left(u_{1}^{\prime}\right)_{x_{1}} u_{x_{2}}^{2}-u_{x_{1}}^{2}\left(u_{1}^{\prime}\right)_{x_{2}}-u_{x_{2}}^{1}\left(u_{2}^{\prime}\right)_{x_{1}}\right) d x & =\int_{\Omega} R(\mathbf{u}) \mathbf{u}^{\prime} d x \\
& +\int_{\Gamma} P(\mathbf{u}) \mathbf{u}^{\prime} d s .
\end{aligned}
$$

Hence,

$$
d J_{3}(\Omega ; \mathbf{u}) \mathbf{h}=\int_{\Omega} R(\mathbf{u}) \mathbf{u}^{\prime} d x+\int_{\Gamma} P(\mathbf{u}) \mathbf{u}^{\prime}+g_{3}(\operatorname{det} \nabla \mathbf{u}) \mathbf{h} \cdot \mathbf{n} d s .
$$

From system (36), we have for the solution $(\boldsymbol{\lambda}, q)$ of the adjoint system $(41,43)$

$$
0=\int_{\Omega}\left(\left(-v \Delta \mathbf{u}^{\prime}+D \mathbf{u} \cdot \mathbf{u}^{\prime}+D \mathbf{u}^{\prime} \cdot \mathbf{u}+\nabla p^{\prime}\right) \cdot \boldsymbol{\lambda}-\left(\operatorname{div} \mathbf{u}^{\prime}\right) \cdot q\right) d x .
$$

Applying Greens formula gives

$$
\begin{aligned}
0 & =\int_{\Omega}\left(\left(-v \Delta \boldsymbol{\lambda}-D \boldsymbol{\lambda} \cdot \mathbf{u}+[D \mathbf{u}]^{t} \cdot \boldsymbol{\lambda}+\nabla q\right) \cdot \mathbf{u}^{\prime}-(\operatorname{div} \boldsymbol{\lambda}) \cdot p^{\prime}\right) d x \\
& -\int_{\Gamma} \mathbf{u}^{\prime}(q \mathbf{n}-(\mathbf{u} \cdot \mathbf{n}) \boldsymbol{\lambda}-v D \boldsymbol{\lambda} \cdot \mathbf{n}) d s-\int_{\Gamma}\left(-p^{\prime} \mathbf{n}+v D \mathbf{u}^{\prime} \cdot \mathbf{n}\right) \boldsymbol{\lambda} d s .
\end{aligned}
$$

Since $(\boldsymbol{\lambda}, q)$ and $\left(\mathbf{u}^{\prime}, p^{\prime}\right)$ satisfy $(41,43)$ and $(36,38)$ respectively, we have

$$
\int_{\Omega} R(\mathbf{u}) \cdot \mathbf{u}^{\prime} d x=-\int_{\Gamma_{f}}(v D \lambda \cdot \mathbf{n}-q \mathbf{n}) \mathbf{u}^{\prime} d s .
$$

The term $(q \mathbf{n}) \mathbf{u}^{\prime}$ in (50) vanishes on $\Gamma_{f}$, due to Lemma 4.2. Hence, using (38), we obtain the Eulerian derivative from (49):

$$
d J_{3}(\Omega ; \mathbf{u}) \mathbf{h}=\int_{\Gamma_{f}}\left(g_{3}(\operatorname{det} \nabla \mathbf{u})+D(\mathbf{u}-\mathbf{g}) \cdot \mathbf{n} \cdot(v D \boldsymbol{\lambda} \cdot \mathbf{n}-P(\mathbf{u}))\right) \mathbf{h} \cdot \mathbf{n} d s .
$$

Since the mapping $\mathbf{h} \mapsto d J_{3}(\mathbf{u}, \Omega) \mathbf{h}$ is linear and continuous, we get the expression for the shape gradient (46). 


\section{Algorithmic realization and numerical examples}

In this section, we present the algorithm that we use to solve the shape optimization problems. Furthermore, computational results to the selected test problems are presented. We denote by $\Omega_{0}, \Omega_{f}$ the initial and the final shapes respectively. Using the gradient information from the previous section, the domain $\Omega$ is deformed according to the following algorithm.

\subsection{The boundary variation algorithm}

As announced in Remark 4.1, we now explain how to obtain the globally defined field $\mathbf{h}$ from data specified on $\Gamma_{f}$ only. These data will be taken as one of the negative gradients according to (44)-(46). For the geometries depicted in Figure 2, only one of the coordinates of $\nabla J_{i} \mathbf{n}$ is nontrivial. Thus Algorithm 1 defines the deformation field $\mathbf{h}$

Algorithm 1 The boundary variation algorithm

1. Choose initial shape $\Omega_{0}$;

2. Compute the state system and the adjoint system, then evaluate the descent direction $\mathbf{h}_{k}$ by using

$$
\begin{aligned}
-\Delta \mathbf{h}+\mathbf{h} & =0 \text { in } \Omega, \\
\frac{\partial \mathbf{h}}{\partial \mathbf{n}} & =-\nabla J \mathbf{n} \text { on } \Gamma_{f}, \\
\mathbf{h}=0 & \text { on } \Gamma_{\text {in }} \cup \Gamma_{w} \cup \Gamma_{\text {out }},
\end{aligned}
$$

with $\Omega=\Omega_{k}$;

3. Set $\Omega_{k+1}=\left(I d+t_{k} \mathbf{h}_{k}\right) \Omega_{k}$ where $t_{k}$ is a positive scalar.

over the entire domain. It provides a decent direction for the cost functional $J$ :

$$
d J(\mathbf{u}, \Omega) \mathbf{h}=\int_{\Gamma_{f}} \nabla J \mathbf{n} \cdot \mathbf{h} d s=-\int_{\Omega}|\nabla \mathbf{h}|^{2}+|\mathbf{h}|^{2} d x<0 .
$$

Remark 5.1. In the above form, Algorithm 1 does not provide for the inequality constraints in $\mathscr{U}_{\text {ad }}$. To realize these constraints, a penality approach is used. For a positive penalty constant $\mu$, we consider the penalized functional

$$
\mathfrak{F}(\mathbf{u}, \Omega, \mu)=J(\mathbf{u}, \Omega)+\mu \mathscr{E}(\Omega)
$$

where $\mathscr{E}(\Omega): \mathbb{R}^{2} \mapsto \mathbb{R}^{1}$, defined as

$$
\mathscr{E}(\Omega)=\int_{\Omega}\left[\max \left(\mathscr{E}_{u}\left(x_{1}, x_{2}\right), 0\right)+\min \left(\mathscr{E}_{l}\left(x_{1}, x_{2}\right), 0\right)\right] d x,
$$

describes the constraints on admissible shapes $\Omega$. Here $\mathscr{E}_{u}\left(x_{1}, x_{2}\right)$ and $\mathscr{E}_{l}\left(x_{1}, x_{2}\right)$, denote the upper and lower constraints on the shape, respectively. If $\mathscr{E}$ and $J$ are shape 
differentiable, then there exist shape gradients $\nabla \mathscr{E}, \nabla J$ of $\mathscr{E}(\Omega)$ and $J(\mathbf{u}, \Omega)$, respectively. Consequently, the Eulerian derivative of $\mathfrak{F}$ with respect $\Omega$ in the direction $\mathbf{h}$ reads

$$
d \mathfrak{F}(\mathbf{u}, \Omega, \mu) \mathbf{h}=d J(\mathbf{u}, \Omega) \mathbf{h}+\mu d \mathscr{E}(\Omega) \mathbf{h},
$$

where

$$
d \mathscr{E}(\Omega) \mathbf{h}=\int_{\Gamma_{f}}\left[\max \left(\mathscr{E}_{u}\left(x_{1}, x_{2}\right), 0\right)+\min \left(\mathscr{E}_{l}\left(x_{1}, x_{2}\right), 0\right)\right] \mathbf{h} \cdot \mathbf{n} d s
$$

As a result, the computational step in (51-53) is replaced by

$$
\begin{aligned}
-\Delta \mathbf{h}_{k}+\mathbf{h}_{k} & =0 \text { in } \Omega_{k}, \\
\frac{\partial \mathbf{h}_{k}}{\partial \mathbf{n}} & =-\nabla J_{c} \mathbf{n} \text { on } \Gamma_{f}, \\
\mathbf{h}_{k} & =0 \text { on } \Gamma_{\text {in }} \cup \Gamma_{w} \cup \Gamma_{\text {out }},
\end{aligned}
$$

where $\nabla J_{c} \mathbf{n}=\left[\nabla J \mathbf{n}+\mu\left[\max \left(\mathscr{E}_{u}\left(x_{1}, x_{2}\right), 0\right)+\min \left(\mathscr{E}_{l}\left(x_{1}, x_{2}\right), 0\right)\right] \mathbf{n}\right]$.

The constraint $\left|\alpha^{\prime}\right| \leq L_{i}, i=1,2$, is not directly enforced by the above algorithm and therefore possible boundary oscillations can occur. The extension of $\mathbf{h}$ on the basis of (54) is also regularizing. If the Neumann boundary condition in (54) is replaced by a Dirichlet condition, then the regularization is insufficient and undesired oscillations of the shapes occur.

\subsection{Test case 1: flow in a channel with a bump}

We consider the optimization of a flow in a channel with a bump as shown in Fig.1(a). The dimensions of the channel are as follows, $-1<x_{1}<1$ and $-1<x_{2}<1$ with a bump on the upper wall extending from $x_{1}=-0.5$ to $x_{1}=0.5$, i.e., $a=-0.5, b=0.5$, $\alpha_{0}=\alpha_{1}=1$, in $\mathscr{U}_{a d}, \mu=10^{3}$ and $\mathbf{g}=\left(2.5\left(x_{2}+1\right)\left(1-x_{2}\right), 0\right)$. Moreover, we set $\alpha_{\min }\left(x_{1}\right)=0.5$ and $\alpha_{\max }\left(x_{1}\right)=1.5$ as parameters in Figure 2 (a). As a consequence, $\mathscr{E}_{u}\left(x_{1}, x_{2}\right)$ and $\mathscr{E}_{l}\left(x_{1}, x_{2}\right)$ in $(54)$ are given by

$$
\mathscr{E}_{u}\left(x_{1}, x_{2}\right)=-1.5+x_{2} \text { and } \mathscr{E}_{l}\left(x_{1}, x_{2}\right)=-0.5+x_{2}
$$

The computational domain is discretized by triangular elements generated by a bidimensional anisotropic mesh generator. $R e$ is set to 50 and the Navier-Stokes equations are solved using a Picard type iteration. The velocity field is depicted in Figure 3. The flow field pattern (Figure 3) possesses a vortex in the bump region of the computational domain. Its reduction/minimization by using the control boundary $\left(\Gamma_{f}\right)$ is our goal.

Remark 5.2. The motion of $\Gamma_{f}$ is modeled explicitly using boundary nodes which are connected by line segments. These nodes are moved using the deformation field $\mathbf{h}$ computed in (51-53). This field is chosen in accordance to the special parametrization 


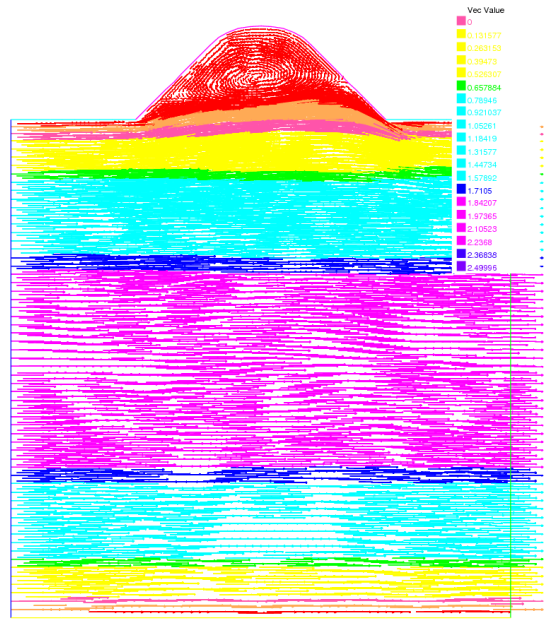

(a) Un zoomed

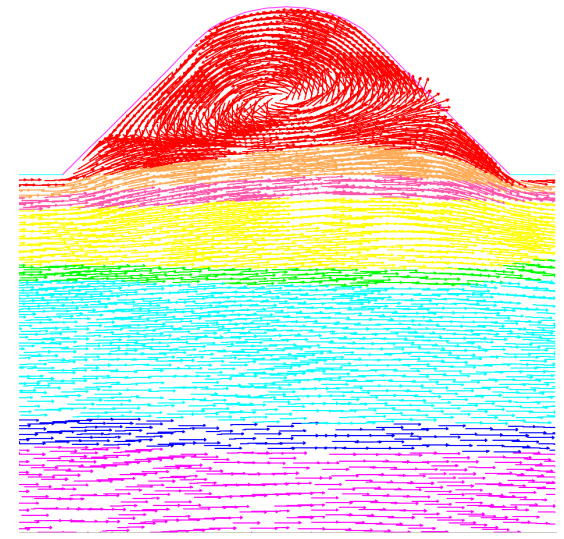

(b) Zoomed

Figure 3: Vector plot of velocity vectors $u_{1}$ and $u_{2}$

of shapes of admissible domains. With reference to Figure 2 (a), $\mathbf{h}$ is chosen as $\mathbf{h}=$ $\left(0, h_{2}\right)$ where

$$
-\Delta h_{2}+h_{2}=0 \text { in } \Omega, \frac{\partial h_{2}}{\partial \mathbf{n}}=-\nabla J_{c} n_{y} \text { on } \Gamma_{f}, h_{2}=0 \text { on } \Gamma_{\text {in }} \cup \Gamma_{w} \cup \Gamma_{\text {out }},
$$

meaning that only $\Gamma_{f}$ varies.

The choice of boundary condition for this example ensures that the solution of (4-5) has a parabolic profile if $\Omega$ is a square. More precisely, if $\Omega$ is a square $[-1,1] \times[-1,1]$, the solution is given by:

$$
\left\{\begin{array}{l}
u\left(x_{1}, x_{2}\right)=\left(2.5\left(x_{2}+1\right)\left(1-x_{2}\right), 0\right), \\
p\left(x_{1}, x_{2}\right)=\frac{5}{R e}\left(1-x_{1}\right) .
\end{array}\right.
$$

\subsubsection{Shape optimization with $\operatorname{cost} J_{1}$}

The desired state is chosen as $\mathbf{u}_{d}=\left(2.5\left(x_{2}+1\right)\left(1-x_{2}\right), 0\right)$. With this choice of target flow, the functional $J_{1}$ vanishes at the optimal domain, which in this case is known to be a square. We start the algorithm with initial flow as in Figure 3. The $\mathbf{H}^{1}(\Omega)$ norm of $\mathbf{h}$ together with the maximum value of $\mathbf{h}$ on the control boundary are used as the stopping criteria for the algorithm. As expected, after optimization, we obtain an axis-parallel flow field on a square geometry $\left(\Omega_{f}\right)$. The value of the cost $J_{1}$ on the initial geometry is 0.118577 while that on the final geometry is $1.97727 \times 10^{-11}$. A plot of the history of the three cost functions during the minimization process according to $J_{1}$ results in Figures 4 (a-c). From these figures, we see that as the number of iterations increases, both cost $J_{1}$ and $J_{3}$ decrease while $J_{2}$ increases. This means that the optimal geometry which minimizes both $J_{1}$ and $J_{3}$ is not the optimal one for $J_{2}$. 


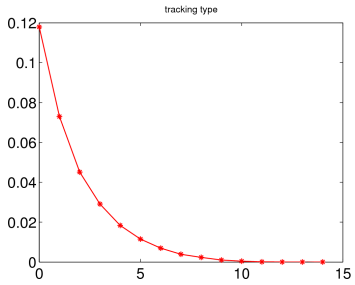

a) History of $J_{1}$

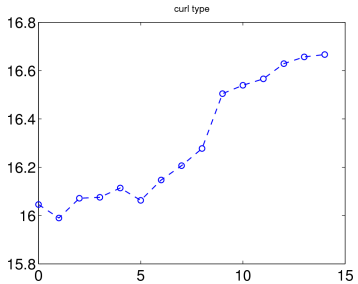

b) History of $J_{2}$

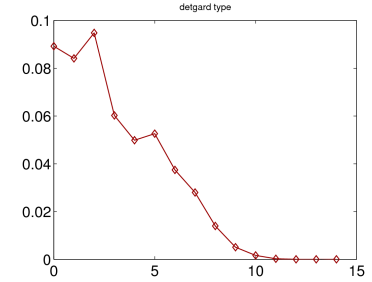

c) History of $J_{3}$

Figure 4: History of three cost functionals during minimization of tracking type cost

\subsubsection{Shape optimization with $\operatorname{cost} J_{2}$}

The results from the previous subsubsection indicate that the optimal geometry which minimizes $J_{2}$ is not a square. In this case initialization is made with geometry $\Omega_{0}$ as

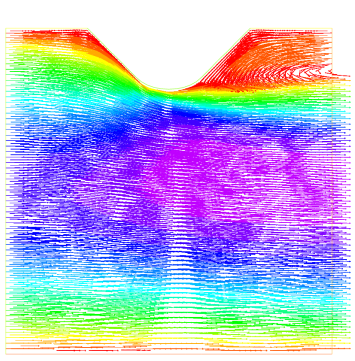

(a) Initial flow field

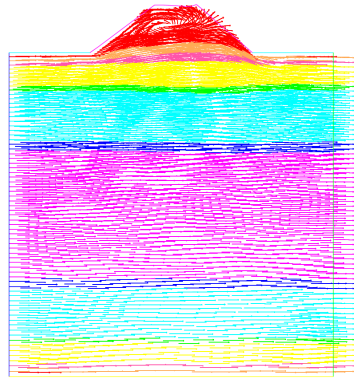

(b) Final flow field

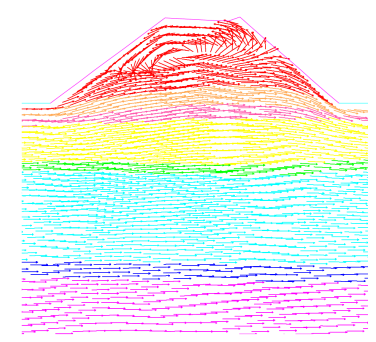

(c) Zoomed field

Figure 5: Initial geometry, flow field and final flow field with $J_{2}$

shown in Figure 5 (a) and the value of the cost on $\Omega_{0}$ is 32.69. After 14 iterations, the value of the cost on the final design is 16.0 which gives a relative reduction of $50.87 \%$ of the initial cost. From Figure $5(b-c)$, we see that although we have reduced the value of the cost functional $J_{2}$, a vortex is created. From a physical point of view, energy is applied to overcome the effect of the wall where no slip boundary condition holds. This energy loss is proportional to the normal gradient of the tangential velocity component at the wall. Compared to our example, this gradient is reduced by separation giving results shown in Figure 5. Hence, this shows that in this particular example, cost functional $J_{2}$ seems not to be a good candidate for vortex reduction.

\subsubsection{Shape optimization with $\operatorname{cost} J_{3}$}

Similar results as in the case of $J_{1}$ are obtained when $J_{3}$ is minimized.

Remark 5.3. We remark here that the optimal geometry obtained when using the tracking type cost functional depends on how we define the desired flow $\mathbf{u}_{d}$. A different choice other than the parabolic flow profile will yield a different optimal geometry. 


\subsection{Test case 2: flow in a channel with an obstacle}

Here the goal is the reduction of the vortex shedding behind an obstacle placed in a parallel channel by changing the shape of one of its boundaries. The cost criteria are again the three cost functionals introduced in the previous sections. In this example, the exact solution to the flow problem is not known. Due to the fact that the cost functionals are non-convex, the initialization of the algorithm can be important. A direct numerical simulation for different geometries is performed and the value of each of the three cost functionals on these geometries is computed. The geometry which gives the least cost will be used as the initial guess for the boundary variation algorithm.

\subsubsection{Computational geometries and direct numerical simulation}

The dimensions of the channel in Figure 2 (problem 2) are set as follows, $L=2$, $H=1, d=0.3, e=0.7, \alpha_{\min }\left(x_{2}\right)=0.54$ and $\alpha_{\max }\left(x_{2}\right)=0.95$. Three of the possible initialization configurations for $\Gamma_{f}$ (refer to Figure 2, problem 2 ) are considered. The resulting computational domains are then discretized by triangular elements generated by a bi-dimensional anisotropic mesh generator. The boundary conditions are set as in (5) where $\mathbf{g}=\left(1.2\left(0.5-x_{2}\right)\left(x_{2}+0.5\right), 0\right)$. The Reynold's number $R e$ is set equal to 120. The following flow field patterns shown in Figure 6 are obtained. After computation of the numerical solution in each of the three cases, the

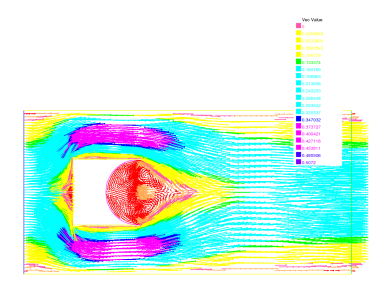

(a) Geom 1

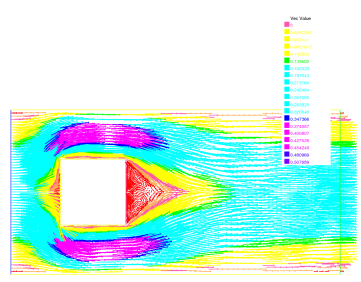

(b) Geom 2

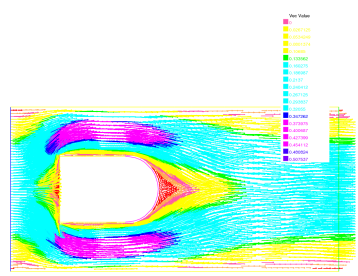

(c) Geom 3

Figure 6: Vector plots of flow field patterns

values of each of the three cost functionals are computed and reported in Table 1. In each column, the least value of the cost obtained after evaluation on each of the

Table 1: The values of the cost functionals on the three geometries

\begin{tabular}{|c|c|c|c|}
\hline Geom & Cost $J_{1}$ (Tracking) & Cost $J_{2}($ Curl) & Detgrad cost $J_{3}$ \\
\hline 1 & 0.0567474 & 3.96193 & 0.329811 \\
2 & $\mathbf{0 . 0 5 6 5 5 4 9}$ & $\mathbf{3 . 9 5 4 3 5}$ & 0.327914 \\
3 & 0.0577802 & 4.25205 & $\mathbf{0 . 3 1 0 8 6 7}$ \\
\hline
\end{tabular}

three geometries is marked with bold font, e.g., for the tracking type cost, the second geometry gives the least value of this cost functional and so on. Now that we have some idea of where the optimal geometry for each of the three cost functional lies, the task is to use a numerical optimization procedure to find the optimal shapes that minimize each of the three cost functionals. In the following subsections, we set 
$\mathscr{E}_{u}\left(x_{1}, x_{2}\right)=-0.95+x_{1}, \mathscr{E}_{l}\left(x_{1}, x_{2}\right)=-0.54+x_{1}$ and $\mu=10^{3}$. Furthermore, we chose $\mathbf{h}=\left(h_{1}, 0\right)$, where $-\Delta h_{1}+h_{1}=0$ in $\Omega, \frac{\partial h_{1}}{\partial \mathbf{n}}=-\nabla J_{c} n_{x}$ on $\Gamma_{f}, h_{1}=0$ on $\Gamma_{\text {in }} \cup \Gamma_{w} \cup \Gamma_{\text {out }}$, meaning that only $\Gamma_{f}$ varies.

\subsubsection{Optimization with Tracking $\operatorname{cost} J_{1}$}

The desired state is chosen as $\mathbf{u}_{d}=\left(0.07\left(0.5-x_{2}\right)\left(x_{2}+0.5\right), 0\right)$. This choice of the desired state is motivated by the fact that we want to suppress the vortex in the flow around the obstacle. The optimization is initialized from the geometry that gives a minimum cost after direct numerical simulation, (c.f. Table 1). The following results are obtained. In Figure 7, we show the flow field on the final geometry obtained after

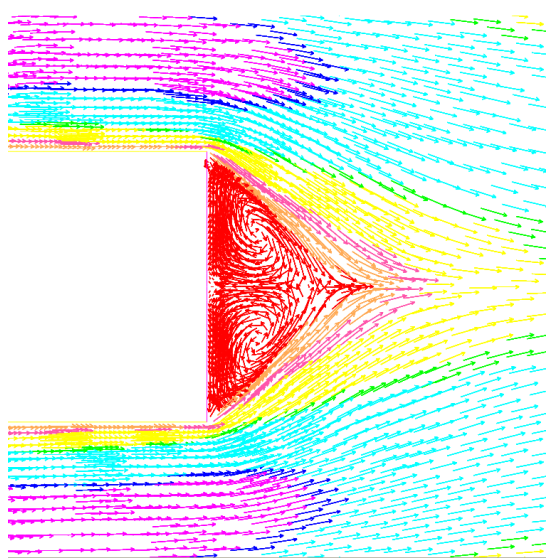

(a) Initial flow field

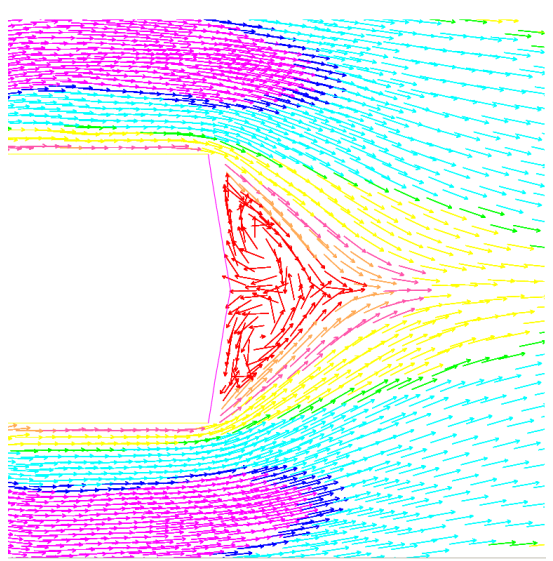

(b) Flow field on final geometry

Figure 7: Zoomed velocity field on initial and optimal geometry

17 iterations. The value of the cost on the initial geometry is found to be 0.0577918 while that on the final geometry is 0.0564491 . Although the cost has been reduced by $2.3 \%$ with respect to the initial value of the cost, the flow field on the final geometry still possess a vortex. We remark that the optimization using this cost depends upon the definition of the desired state, i.e., different desired state values $\mathbf{u}_{d}$ yield different optimal shapes.

\subsubsection{Optimization with curl type $\operatorname{cost} J_{2}$}

In this case we start with a discretized geometry with an initial flow as in Figure 6 (b). In Figure 8, we show the flow field on the final geometry. The value of the cost on the initial geometry is 3.95709 while that on the final geometry is 3.91294 . This gives a relative reduction of $1.1137 \%$ in the value of the cost. However, small vortices are still visible in the region marked by bold circles in Figure 8. 


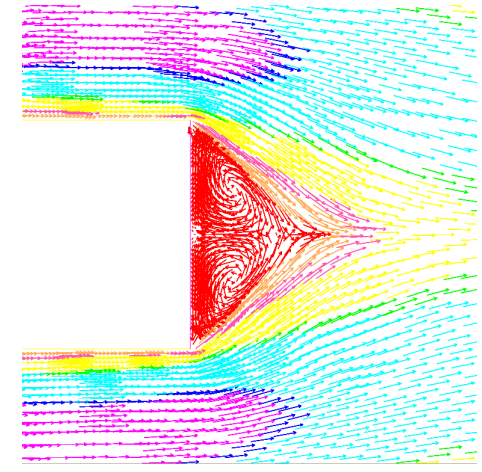

(a) Initial flow field

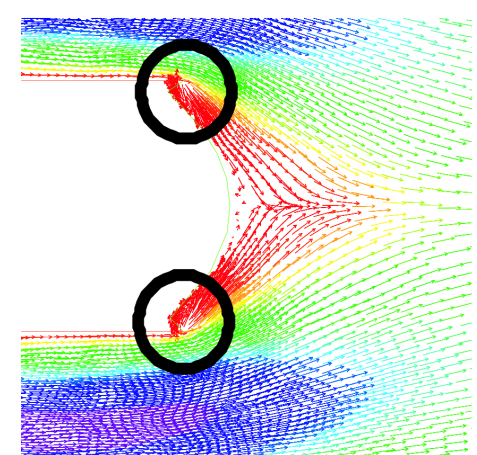

(b) Flow field on final geometry

Figure 8: Zoomed velocity field on initial and optimal geometry

\subsubsection{Optimization with cost $J_{3}$}

We start the computation with the initial geometry $\Omega_{0}$ with a flow as shown in Figure 6(c). The value of the cost $J_{3}$ on $\Omega_{0}$ is found to be 0.311156 (c.f table 1) while that

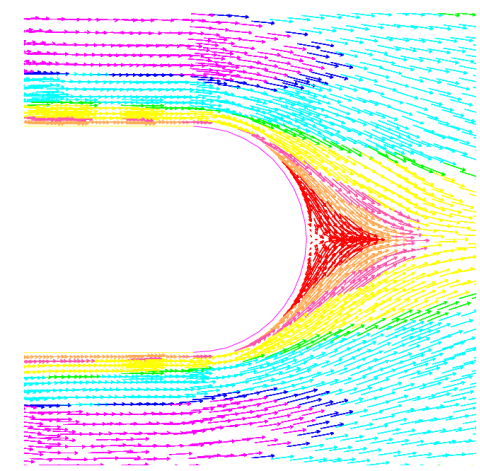

(a) Initial flow field

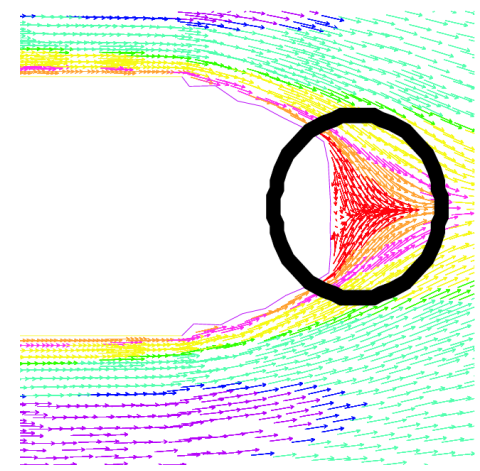

(b) Flow field on final geometry

Figure 9: Zoomed velocity field on initial and optimal geometry

on $\Omega_{\text {opt }}$ (Figure $9(\mathrm{~b})$ ) is 0.2994 . This gives a relative reduction of $3.7782 \%$ in the value of the cost after 17 iterations. A further zoom of the final flow field in a region marked with a circle in Figure 9 indicated no visual presence of vortices in the flow field. These results from minimization of $J_{3}$ suggest that cost $J_{3}$ performs better than to $J_{1}$ (see Figure $7(\mathrm{~b})$ ) and $J_{2}$ (see Figure $8(\mathrm{~b})$ ) in reducing the vortex in the region behind the obstacle.

\subsection{Test case 3 : an irrotational flow in a channel with a bump}

In this example, we consider a steady, incompressible, viscous irrotational flow in a channel $-1<x_{1}<1$ and $-1<x_{2}<1$ having a bump on the upper wall extending from 
$x_{1}=-0.5$ to $x_{1}=0.5$. We choose $\mathbf{h}$ as well as the constraints $\mathscr{E}_{l}\left(x_{1}, x_{2}\right)$ and $\mathscr{E}_{u}\left(x_{1}, x_{2}\right)$ as in test case 1 . Here the flow under consideration is assumed to be irrotational flow, i.e., curl $\mathbf{u}=0$. This guarantees existence of a velocity potential $\Phi$, which is related to the velocity components by $u_{1}=\frac{\partial \Phi}{\partial x_{1}}$ and $u_{2}=\frac{\partial \Phi}{\partial x_{2}}$. In order to construct an exact irrotational velocity field that solves (4) on $\Omega=(-1,1) \times(-1,1)$, we choose $\Phi=$ $-2 x_{1} x_{2}$ such that

$$
\mathbf{u}=\left(-2 x_{2},-2 x_{1}\right) .
$$

This results in the following boundary conditions

$$
\begin{cases}\mathbf{u}=\left(2,-2 x_{1}\right) \text { on } \Gamma_{1}, & \mathbf{u}=\left(-2 x_{2},-2\right) \text { on } \Gamma_{2}, \\ \mathbf{u}=\left(-2,-2 x_{1}\right) \text { on } \Gamma_{3} \cup \Gamma_{f}, & \mathbf{u}=\left(-2 x_{2}, 2\right) \text { on } \Gamma_{4} .\end{cases}
$$

The body forces $\mathbf{f}$ are chosen in such a way that if $\Omega$ is a square $(-1,1) \times(-1,1)$, then the exact solution of (4), (57) is given by (56). Hence, we choose $\mathbf{f}=\left(4 x_{1}, 4 x_{2}\right)$. The value of the Reynolds number is set to 50 and system (4) together with the above

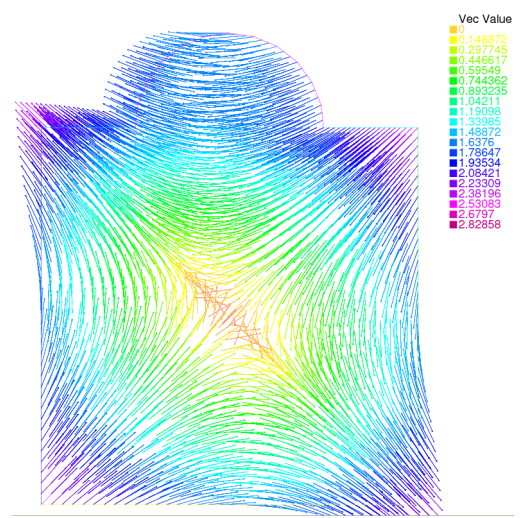

Figure 10: Flow on initial geometry

data is solved. This results in the flow field on the initial geometry depicted in Figure 10. This example is chosen to further highlight the different behavior of the three cost functionals. For this irrotational flow, a proper choice of cost functional for vortex reduction should have the property that it is insensitive with respect to changes of the domain. In particular, an iterative algorithm is expected to stop after the first iteration from any initial choice $\Omega_{0}$. However as we shall see below, this is not the case. There is still a clear distinction between the optimal domains corresponding to the 3 cost functionals.

\subsubsection{Shape optimization with cost $J_{1}$}

The desired flow field is chosen as $\mathbf{u}_{d}=\left(-2 x_{2},-2 x_{1}\right)$ and the cost functional $J_{1}$ is minimized. The algorithm is initialized with the geometry shown in Figure 10. A 
similar stopping criterion as in the previous examples is set. The cost $J_{1}$ is found to be sensitive with respect to changes of the domain. In Figure 11, we display the results obtained after optimization. The final geometry is obtained after 20 iterations. The

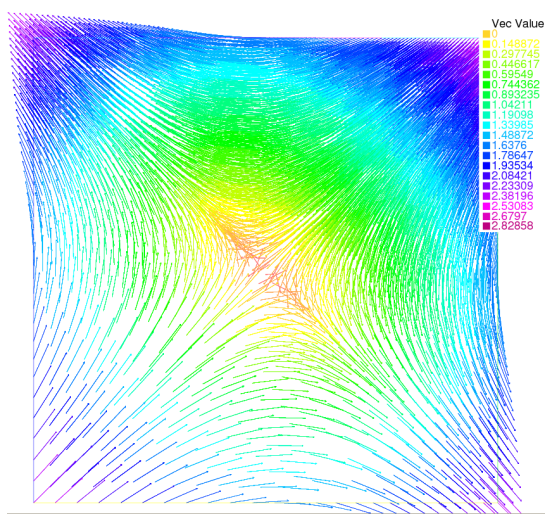

(a) Flow field on final geometry

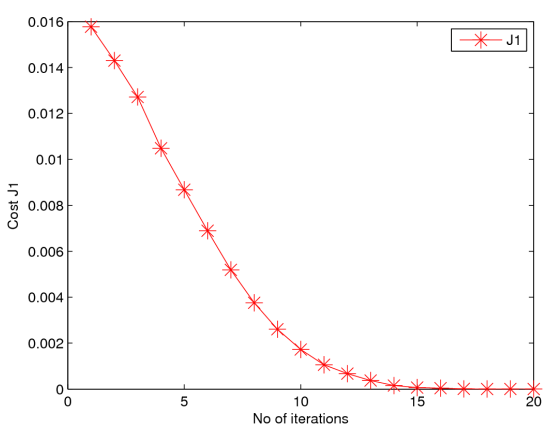

(b) History of cost $J_{1}$

Figure 11: Final geometry and history of cost $J_{1}$

value of the cost $J_{1}$ on the initial geometry is 0.01577 while that on the final geometry is $3.731 \times 10^{-9}$. As expected in view of $\mathbf{u}_{d}$ as the desired state in the tracking functional, the final geometry is a square with an irrotational flow field.

\subsubsection{Shape optimization with $\operatorname{cost} J_{2}$}

The cost $J_{2}$ is found to be sensitive with respect to changes of the domain. In Figure

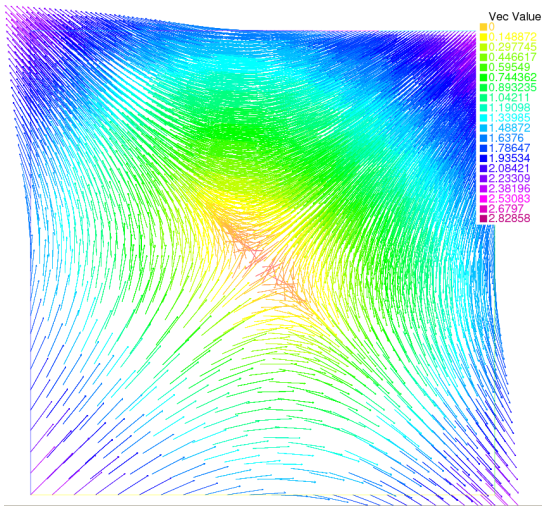

(a) Flow field on final geometry

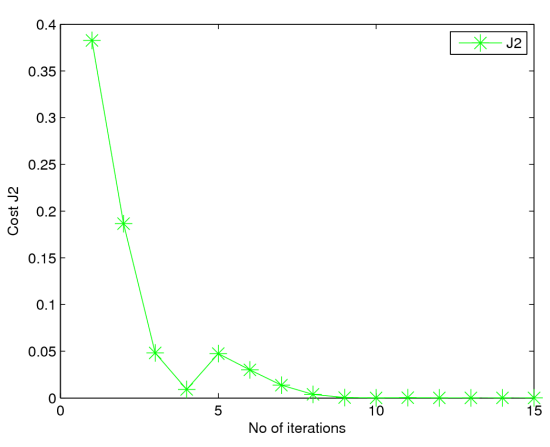

(b) History of cost $J_{2}$

Figure 12: Final geometry and history of cost $J_{2}$

12 (a), we depict the flow field on $\Omega_{f}$, and in ( 12 (b)), the history of cost $J_{2}$. The final geometry is obtained after 15 iterations. The value of the cost $J_{2}$ on the initial geometry 
is 0.3827 while that on the final geometry is $3.21 \times 10^{-6}$. As expected in view of the boundary conditions (57), the final geometry is a square with an irrotational flow field.

\subsubsection{Shape optimization with $\operatorname{cost} J_{3}$}

The cost $J_{3}$ is found to be insensitive with respect to domain changes, i.e., we observe stagnation after 1 iterations and we stop the algorithm. This is the case since the cost as well as the shape gradient are already zero for the given flow field. Thus the deformation field required to change the geometry is zero just after the first iteration, and the returned geometry is the same as the initial geometry with the value of the cost of order $10^{-8}$.

\subsection{Conclusions}

Our results confirm that the choice of cost functional is important for vortex reduction in fluid dynamics. The Galilean invariant cost functional $J_{3}$ should be preferred over the commonly used functionals $J_{2}$ and $J_{1}$.

Remark 5.4. In case of 3-dimensional flows, it appears that further considerations are necessary on how to design a practical cost functional to be used for vortex reduction. Research in [3] shows that $\nabla \mathbf{u}$ has one real and two complex conjugate eigen values for the regions in space where

$$
\Delta=\left(\frac{Q}{3}\right)^{3}+\left(\frac{\operatorname{det} \nabla \mathbf{u}}{2}\right)^{2}>0
$$

where $Q=\frac{1}{2}\left(|\tilde{\Omega}|^{2}-|S|^{2}\right), \tilde{\Omega}=\frac{1}{2}\left(\nabla \mathbf{u}+(\nabla \mathbf{u})^{T}\right)$, and $\tilde{\Omega}=\frac{1}{2}\left(\nabla \mathbf{u}-(\nabla \mathbf{u})^{T}\right)$. Thus spatial domains where $\Delta>0$ are candidates for local instantaneous stirring. Therefore, it could be forth-while to investigate the three dimensional case with det $\nabla \mathbf{u}$ in (3) replaced by $\Delta$.

\section{Acknowledgment}

The authors would like to thank Prof. Brenn from the Technical University of Graz for a very helpful discussion.

\section{References}

[1] F. Abergel and R. Temam. On some control problems in fluid mechanics. Theoretical and Computational Fluid Dynamics, 1(6):303-325, 1990.

[2] M. Bach, C. Constanda, G. C. Hsiao, A. M. Sandig, and P. Werner. Analysis, Numerics and Applications of Differential and Integral Equations, vol. 379. Research Notes in Mathematics Series. Addison Wesley Longman Limited, USA, 1998. 
[3] H. M. Blackburn, N. N. Mansour, and B. J. Cantwell. Topology of fine-scale motions in turbulent channel flow. Journal of Fluid Mechanics, 310:269-292, 1996.

[4] S. Boisgérault and J. P. Zolésio. Shape derivative of sharp functionals governed by navier-stokes flow. In W. Jäger, J. Necăs, O. John, K. Najzar, and J stará, editors. Partial Differential Equations: Theory and Numerical Solution, pages 49-63. Chapman \& Hall/CRC Reseach Notes in Mathematics, 1993.

[5] D. Chenais. On the existence of a solution in a domain identification problem. $J$. Math. Anal and Appl., 52:189-219, 1975.

[6] M. S. Chong, A. E. Perry, and B. J. Cantwell. A general classification of threedimensional flow fields. Physics of Fluids, 2(5):765-777, May 1990.

[7] M. C. Delfour and J. P. Zolésio. Shapes and geometries: analysis, differential calculus, and optimization. Society for Industrial and Applied Mathematics, Philadelphia, PA, USA, 2001.

[8] V. Girault and P. A. Raviart. Finite Element Methods for Navier-Stokes. SpringerVerlag, Berlin, 1986.

[9] P. Grisvard. Elliptic Problems in Nonsmooth Domains, Monographs and Studies in Mathematics,vol. 24. Pitman, Massachusetts, 1985.

[10] M. Gunzburger. Flow Control. IMA 68, Editor M.D. Gunzburger. Springer, Berlin, 1995.

[11] G. Haller. Lagrangian structures and the rate of strain in a partition of twodimensional turbulence. Physics of Fluids, 13(11):3365-3385, 2001.

[12] G. Haller. An objective definition of a vortex. Journal of Fluids Mechanics, 525:1-26, 2005.

[13] J. Haslinger and R. A. E. Mäkinen. Introduction to Shape Optimization: Theory, Approximation, and Computation. Society for Industrial and Applied Mathematics, Philadelphia, PA, USA, 2003.

[14] A. Henrot and Y. Privat. What is the optimal shape of a pipe? Archive for Rational Mechanics and Analysis, 196(1):281-302, 2009.

[15] J. G. Heywood, R. Rannacher, and S. Turek. Artificial boundraies and flux and pressure conditions for the incompressible navier-stokes equations. International Journal for Numerical Methods in Fluids, 22(5):325-352, 1996.

[16] M. Hintermüller, K. Kunisch, Y. Spasov, and S. Volkwein. Dynamical systems based optimal control of incompressible fluids. Int. J. Numer. Methods in Fluids, 4:345-359, 2004.

[17] J. Jeong and F. Hussain. On the identification of a vortex. Journal of Fluids Mechanics, 285:69-94, 1995. 
[18] W. Kaplan. Ordinary differential Equations. Addison-Wesley, Reading, Mass, 1967.

[19] K. Kunisch and B. Vexler. Optimal vortex reduction for instationary flows based on translation invariant cost functionals. SIAM J. Control Optim., 46(4):13681397, 2007.

[20] D. Martinec. Lecture notes on continuum mechanics. [pdf document] retrieved from lecture notes online. Web site: http://geo.mff.cuni.cz/vyuka/MartinecContinuumMechanics.pdf.

[21] M. Moubachir and J.P. Zolésio. Moving Shape Analysis : Applications to Fluid Structure Interactions. Chapman and Hall, USA, 2006.

[22] J. Sokolowski and J. P. Zolésio. Introduction to Shape Optimization. Shape Sensitivity Analysis. Springer-Verlag, 1992.

[23] R. Temam. Navier Stokes Equations: Theory and Numerical Analysis (Studies in Mathematics and its Applications 2. North-Holland, 1977. 